\title{
Passive flexibility effect on oscillating foil energy harvester
}

Wendi Liu , Qing Xiao and Qiang Zhu

NOTICE: this is the author's version of a work that was accepted for publication in the AIAA Journal. Changes resulting from the publishing process, such as final peer review, editing, corrections, structural formatting, and other quality control mechanisms may not be reflected in this document. Changes may have been made to this work since it was submitted for publication. A definitive version was subsequently published in the AIAA Journal, DOI: 10.2514/1.J054205. 


\title{
Passive flexibility effect on oscillating foil energy harvester
}

\author{
Wendi Liu ${ }^{1}$ and Qing Xiao ${ }^{2,3}$ \\ University of Strathclyde, Glasgow, G4 OLZ, UK \\ Qiang $\mathrm{Zhu}^{4}$ \\ University of California, San Diego,La Jolla, CA 92093, USA
}

It is well-known that structural flexibility enhances the performance of flapping foil propellers. There is, however, much less knowledge about the effect of deformability on the flow energy extraction capacity of flapping foils. Following our recent work on an oscillating foil energy harvesting device with prescribed foil deformations ${ }^{1}$, we investigate the fully-coupled dynamics of a flapping foil energy harvester with a passively deformable foil. Towards this end, we computationally study the dynamics of a foil with realistic internal structure (containing a rigid leading edge and a flexible trailing edge with a stiffener) in energy harvesting regime through a fluid-structure interaction scheme. To examine the effect of different levels of flexibility, various materials (ranging from metals such as copper to virtual materials with arbitrary elasticity and density) for the stiffener have been tested. With the virtual materials, the effects of Young's modulus coefficient and density ratio have been studied. Our simulation results show that flexibility around the trailing edge could enhance the overall energy extraction performance. For example, with a copper stiffener, an increase of $32.2 \%$ in efficiency can be reached at high reduced frequency. The performance enhancement is achieved mostly in cases with low Young's modulus coefficient and density ratio. A possible underlying mechanism is that the specific foil deformations in these cases encourage the generation and shedding of vortices from the foil leading edge, which is known to be beneficial to flow energy extraction.

\footnotetext{
${ }^{1} \mathrm{PhD}$ student, Department of Naval Architecture, Ocean and Marine Engineering.

${ }^{2}$ Senior lecturer, Department of Naval Architecture, Ocean and Marine Engineering, AIAA Senior Member.

${ }^{3}$ Corresponding author, Email: qing.xiao@strath.ac.uk.

${ }^{4}$ Associate professor, Department of Structural Engineering.
} 


\section{Nomenclature}

$A=$ sweep area $\left(\mathrm{m}^{2}\right)$

$A R=$ aspect ratio

$c \quad=\quad$ chord length $(\mathrm{m})$

$C_{L}(t)=$ instantaneous lift coefficient

$C_{o p}=$ power coefficient

$c_{\text {pit }}=$ pitching centre $(\mathrm{m})$

$c_{s f}=$ length of metal stiffener $(\mathrm{m})$

$D R=$ density ratio

$E \quad=\quad$ Young's modulus $(\mathrm{Pa})$

$E_{c}=$ Young's modulus coefficient

$f_{0}=$ frequency of flapping wing $(\mathrm{Hz})$

$f_{0.18}=$ frequency of flapping wing under $f^{*}=0.18(\mathrm{~Hz})$

$f_{n} \quad=\quad$ natural frequency of selected material $(\mathrm{Hz})$

$F R=$ natural frequency ratio which defined as $f_{n} / f_{0.18}$

$f^{*}=$ reduced frequency

$g=$ gravitational acceleration $\left(\mathrm{m} / \mathrm{s}^{2}\right)$

$h(t)=$ instantaneous heaving position $(\mathrm{m})$

$h_{0}=$ amplitude of heaving motion (m)

$L \quad=\quad$ lift force $(\mathrm{N})$

$M=\operatorname{moment}(\mathrm{Nm})$

$M_{Z}(t)=\quad$ instantaneous moment coefficient

$p=$ instantaneous power $(\mathrm{W})$

$R e=$ Reynolds number

$S t=$ Strouhal number of foil oscillating

$t \quad=\quad$ instant time $(\mathrm{s})$

$T=$ oscillating period $(\mathrm{s})$

$T_{s f}=$ thickness of metal stiffener (m)

$U_{\infty}=$ free-stream velocity $(\mathrm{m} / \mathrm{s})$

$\beta_{0}=$ the instantaneous non-dimensional trailing edge bending distance normalize by chord relative to local coordinate 
$\beta_{0 \_ \text {amp }}=$ the amplitude of non-dimensional trailing edge bending distance normalize by chord relative to local coordinate

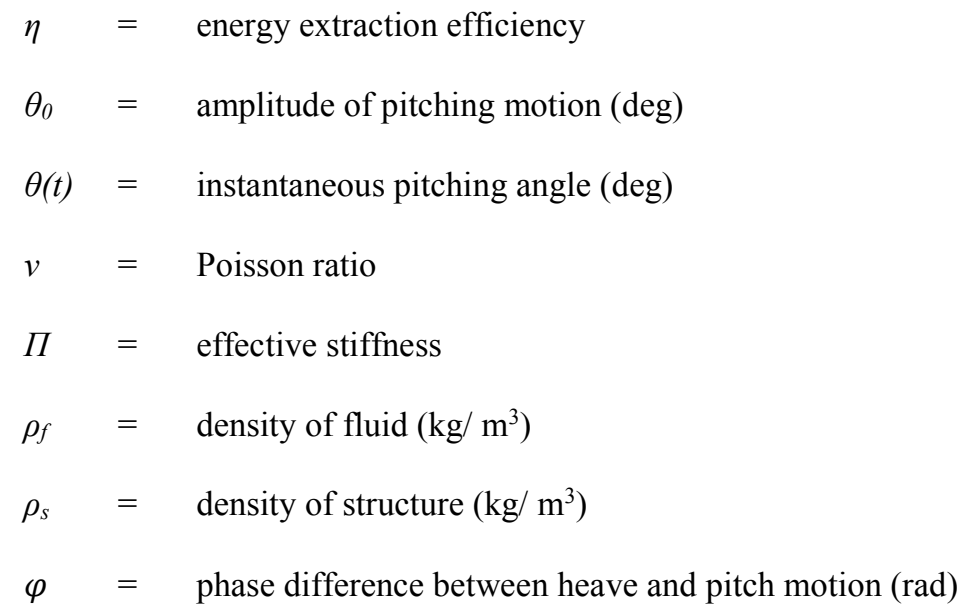

\section{Introduction}

Most of existing tidal current renewable energy devices can be classified into three groups, i.e., horizontal axis turbine, vertical axis turbine and oscillating foil turbine. The bio-inspired tidal energy harvester, an oscillating foil turbine imitating the fish fins or insect wings and utilizing their flapping motions to extract energy from their surrounded fluid, is one of the most novel devices. Compared with the traditional rotating turbine, oscillating foil turbines present several distinguishing characteristics, such as its suitableness in shallow water, mitigation of centrifugal force/stress and low noise during its operation ${ }^{2}$. Since the initial development of McKinney and DeLaurier ${ }^{3}$, in the past few years oscillating foil turbines have been extensively studied because of the growing interests in biomimetics and the improved knowledge about unsteady fluid dynamics involved in flying and swimming motions ${ }^{4}$. The studies about flapping foil energy harvester fall into three categories: fully activated systems with prescribed motions, semi-activated systems with one degree-of-freedom activated and another one induced by fluid dynamic loads, and fully passive systems in which the motions are completely generated by flow-induced instability (i.e. flutter phenomenon) as stated in recent review paper by Xiao and $\mathrm{Zhu}^{2}$. Among these, the fully activated systems are usually used as model systems. By assuming that the foil undergoes certain prescribed motions (in reality these motions can be generated either semi-actively or passively), these model systems are useful in examining the power extraction capacity of the system and the underlying physics. Since the research about flapping foil energy harvesters is still at the beginning stage, most of the existing studies use prescribed foil motions ${ }^{2}$. In fact, in recent studies by Kinsey and Dumas ${ }^{18,}$, a mechanical device has been designed which is capable of undergoing prescribed flapping motions and extract flow energy. In this device the foil motion is driven by a motor, which is activated over part of the period and 
act as a generator in the rest of the periods. However, most existing investigations are limited to rigid oscillating foils.

In nature, many animals deform their bodies or fins either actively or passively to achieve higher thrust efficiency or generate larger transient forces for maneuvering and stabilizing purposes. Insect wings, such as butterfly or bee wings, can generate asymmetry forces by their twisting and bending motions to achieve high time-averaged forces ${ }^{5}$. Combes and Daniel ${ }^{6,7}$ presented a three-dimensional investigation on the stiffness distribution of insect wings in both chordwise and spanwise directions. They observed that the wing stiffness in the spanwise direction is higher than that in the chordwise direction. A sharply decreasing flexural stiffness is observed from wing base to the leading edge, when a large bending moment is applied. Investigations by $\mathrm{Zhu}^{8}$ indicated that the chordwise flexibility could enhance the propulsion efficiency of the wing. Shoele and $\mathrm{Zhu}^{9}$ numerically studied propulsion by flexible ray fins. Similar to insect wings, ray fins have enhanced stiffness around their leading edges, making it easier to control the local angle of attack (AOA) to mitigate leading edge vortices (LEV) formation and shedding. With flexible fin motion, ray fins can significantly reduce the leading edge AOA to enhance propulsion efficiency. On the other hand, when an insect wing is used for lift generation, its strengthened leading edge helps stabilizing and enhancing the LEV and improves its force-generation performance $^{10}$. In fact, it is generally accepted that LEV enhancement is the key mechanism to obtain higher lift force for a flapping wing system at $\operatorname{Re}$ up to $10^{4}{ }^{11}$.

Associated with the flexible features of flapping wings, the system inertia is also found to play a role on the force-generation performance. Daniel and Combes ${ }^{12}$ investigated the inertia effect of flexible insect wings in air (also see Ref. 13), which is usually ignored by previous researchers. Their results indicated that inertia played a primary role in the wing deformation. $\mathrm{Zhu}^{8}$ investigated the density ratio effect on flapping foil performance by taking the inertia and structure dissipation into account. Two different densities of surrounding media were studied, i.e. air and water. It is found that the structure inertia determines the level of deformation when surrounding media has low density, while the fluid load dominates the deformation when the surrounding media has high density. The propulsion efficiency decreases with foil flexibility in a low density medium. However, it increases with flexibility in high density fluid.

In contrast to the numerous investigations about the effects of structural deformability on the forcegeneration performance of flapping foils, the energy harvesting capacity of flexible flapping foils has not been well understood. In our previous study ${ }^{1}$, a two-dimensional oscillating foil at $R e=1.0 \times 10^{6}$ was examined by using a Navier-Stokes solver under turbulent flow condition. In that study, the deformation of the foil was pre- 
specified. According to that study, leading edge flexibility may lead to earlier shedding of LEV, resulting in changes in phase of the instantaneous forces and moments. Subsequently, a $7.68 \%$ increment in energy extraction efficiency is observed when the effective AOA of $10^{\circ}$. This, however, can only be achieved under certain foil deformations that have to be created through active controlling.

In a recent work by Tian et al. ${ }^{14}$, a fluid-structure interaction model with immersed-boundary method was used to study the flow energy extraction by a flexible plate. Four systems were investigated, i.e. rigid plate, flexible plate, flexible leading segment with rigid trailing segment, and rigid plate with active control of leading segment. Their results show an $11.3 \%$ enhancement of energy extraction efficiency by applying active control to the leading edge segment. However, for a passively flexible plate, no performance enhancement was observed.

In the present study, we examine the energy extraction of a passively flexible flapping foil through a fullycoupled fluid-structure interaction study. Unlike the simplified plate model with zero thickness ${ }^{14}$, a more realistic NACA0012 foil shape is used in the present study. In addition, we take into account the internal structure of the foil by using a stiffener to create the structural deformability. This makes our model closer to the real design. In this study, a finite volume method is used, which fully resolves the boundary layer so that it has better accuracy than the immersed-boundary method.

In the rest of the paper, descriptions of the physical problem and the corresponding numerical methods are listed in Section 2. In Section 3, we first consider cases with metal stiffener to examine whether structural flexibility has beneficial effect on energy extraction. The effects of Young's modulus coefficient and density ratio are then studied separately. Finally, conclusions of these studies are listed in Section 4.

\section{Problem Formulation and Numerical Methodology}

\subsection{Problem description}

The present research considers a two-dimensional oscillating energy harvesting system based on a NACA0012 foil whose deformability is determined by a realistic internal structure. As shown in Fig. 1, the incoming flow is uniform with speed $U_{\infty}$. Similar to the study by Kinsey and Dumas ${ }^{15}$, the Reynolds number based on the incoming flow speed and the chord length $c$ of the foil in the current study is fixed as $10^{3}$ so that a laminar flow assumption is used. In applications, there exists three types of design ${ }^{2}$ : systems with forced pitching and heaving motions, systems with forced pitching and induced heaving motions (semi-activated systems), and systems with self-sustained pitching and heaving motions (self-sustained systems). In the current study, we concentrate on the first type $\mathrm{e}^{3,15,16,17,18,19}$ and examine the effects of structural flexibility upon the energy harvesting performance. 


\section{Kinematics}

As demonstrated in Fig. 1, the kinematics of the foil includes the pitch and heave motions specified as:

$$
\begin{gathered}
h(t)=h_{0} \sin \left(2 \pi f_{0} t\right), \\
\theta(t)=\theta_{0} \sin \left(2 \pi f_{0} t+\varphi\right),
\end{gathered}
$$

where $h(t)$ and $\theta(t)$ are the instantaneous heave and pitch motions with the amplitudes of $h_{0}$ and $\theta_{0}$, respectively. $f_{0}$ is the oscillating frequency of the foil. $\varphi$, which has a fixed value of $-\frac{\pi}{2}$ during the current research, is the phase difference between heave and pitch motions. The pitch axis locates at one quarter chord length from the leading edge of the foil (i.e. $c_{\text {pit }} / c=1 / 4$ ).

Following the study of Kinsey and Dumas ${ }^{18}$, the heave amplitude is chosen to be one chord length $\left(h_{0} / c=\right.$ 1). Two pitching amplitudes, $60^{\circ}$ and $73^{\circ}$, are examined.

The oscillating frequency is usually characterized by the reduced frequency $f^{*}$ defined as

$$
f^{*}=\frac{f_{0} c}{U_{\infty}}
$$

\section{Energy extraction performance}

The key parameters to quantify the energy extraction performance of an oscillating foil system are the nondimensional instantaneous power coefficient $C_{o p}$ and the total energy extraction efficiency $\eta$. They are determined by

$$
C_{o p}=\frac{p}{\frac{1}{2} \rho_{f} U_{\infty}^{3} c}=\frac{1}{U_{\infty}}\left[C_{l}(t) \frac{d h(t)}{d t}+c M_{Z}(t) \frac{d \theta(t)}{d t}\right]
$$

and

$$
\eta=\frac{\bar{p}}{\frac{1}{2} \rho_{f} U_{\infty}^{3} A}=\overline{C_{o p}} \frac{c}{A}
$$

where $p$ is the instantaneous power with a time averaged value of $\bar{p} ; \rho_{f}$ is the fluid density and $A$ is the sweep area of the oscillating wing. $C_{l}(t)$ and $C_{m}(t)$ are the instantaneous lift and moment coefficient defined as

$$
\begin{aligned}
C_{l}(t) & =\frac{L}{\frac{1}{2} \rho_{f} U_{\infty}^{2} c}, \\
M_{Z}(t) & =\frac{M}{\frac{1}{2} \rho_{f} U_{\infty}^{2} c^{2}},
\end{aligned}
$$

where $L$ is the lift force and $M$ is the moment of the foil.

Structural design of flexible foils 
To study the effect of structural deformability on the propulsion performance of a flapping wing, Heathcote and Gursul ${ }^{20}$ and Heathcote et al. ${ }^{21}$ developed chordwisely and spanwisely flexible wing models and performed experimental tests. Subsequently, relevant numerical simulations have also been carried out by Shyy et al. ${ }^{11}$, Gordnier et al. ${ }^{22}$ and Chimakurthi et al. ${ }^{23}$. In the present study, we adopt similar internal designs from the above papers and create a foil with chordwise flexibility (Fig. 2 (a)). With a NACA0012 profile, the deformability of this foil is determined by a stiffener with thickness $T_{s f}$ and length $c_{s f}$. The surrounding material, made of polydimethylsiloxane rubber (PDMS) with Young's modulus of $250 \mathrm{kPa}^{21}$, contributes little to the overall structural dynamic properties (stiffness and inertia) of the foil ${ }^{23,24}$. Thus, only the metal stiffener is considered to evaluate the flexible effect. In the present study, the stiffener thickness $T_{s f}$ and length $c_{s f}$ are fixed for all cases as $9 \times 10^{-3} \mathrm{c}$ and $0.75 \mathrm{c}$, respectively.

With the unsteady fluid loads acting on its surface, the flexible part of the foil deforms passively as shown in Fig. 2 (b). The instantaneous non-dimensional trailing edge bending distance relative to the local X coordinate (a local coordinate that coincides with the instantaneous chord of the foil if there is no deformation) is defined as $\beta_{0}=$ displacement $/ c$, whose amplitude is defined as $\beta_{0 \_a m p}$.

The Young's modulus coefficient $E_{c}$ and the density ratio $D R$ are the two key parameters to describe the structural property. Hereby they are defined as

$$
\left\{\begin{array}{c}
E_{c}=\frac{E}{\rho_{f} g c} \\
D R=\frac{\rho_{s}}{\rho_{f}}
\end{array}\right.
$$

where $\rho_{s}$ is the density of the stiffener. Current study is focused on the flexible foil oscillating in water, thus the fluid density $\rho_{f}$ we are aiming to simulate is $1000 \mathrm{~kg} / \mathrm{m}^{3}$.

We note that the stiffness of the structure is often characterized by the effective stiffness $\Pi^{11}$. This parameter describes the elastic bending forces relative to the fluid dynamic forces as:

$$
\Pi=\frac{E T_{s f}^{3}}{12\left(1-v^{2}\right) \rho_{f} U_{\infty}^{2} c^{3}}
$$

where $v$ is the Poisson ratio of the stiffener and $\mathrm{c}$ is the chord length of the foil. The effective stiffness $\Pi$ is widely tested by the experiment of Heathcote \& Gursul ${ }^{20}$ and Heathcote et al. ${ }^{21}$ and numerical simulations from Chimakurthi et al. ${ }^{23}$ and Gordnier et al. ${ }^{24}$.

\subsection{Mathematical formulations and numerical algorithm}


In this study we apply a fluid-structure interaction model by coupling a Navier-Stokes solver with a linear structural model. High computational efficiency is achieved by the application of an implicit dual-time multigrid method for the flow, as well as a modal analysis approach for the structure. The fluid-structure coupling is achieved by sub-iterations. In the fluid part, a cell-centred finite-volume method is used, with the numerical stability enhanced by residual smoothing. The solution of the nonlinear flow equations is accelerated by using a structured multi-grid method and a parallel multi-block approach through MPI. A grid-deformation algorithm (TFI) is developed to interpolate the deformation of the structural boundaries onto the flow grid. Although in the current study we concentrate on a two-dimensional laminar flow problem, the in-house code we have is capable of solving three-dimensional fluid-structure interaction problems as well as flows in high Reynolds number regimes (through turbulence models such as Baldwin-Lomax algebraic model and k- $\omega$ two equation model). The code is also capable of simulating inviscid flows with Euler equations. The detailed formulations and numerical implementation for current study are listed in the following ${ }^{1}$.

\section{Fluid dynamics}

Our fluid solver is based on the following governing equations of unsteady laminar flow

$$
\frac{\partial}{\partial t} \int_{\Omega} \boldsymbol{W} d \Omega+\oint_{\partial \Omega}\left(\boldsymbol{f}-\boldsymbol{f}_{\boldsymbol{\mu}}\right) d \boldsymbol{S}=0
$$

where $\Omega$ is the control volume and $\boldsymbol{S}$ is the boundary surface. The vector $\boldsymbol{W}$ contains the conservative variables.

$$
\boldsymbol{W}=\{\rho, \rho u, \rho v, \rho w, \rho E\}^{T},
$$

where $\rho$ is the fluid density, $u, v$, and $w$ are the three Cartesian velocity components and the flow specific total energy $E$ is given by

$$
E=e+\frac{u_{i} u_{i}}{2}
$$

where $e$ is the internal energy.

The flux tensors $\boldsymbol{f}$ and $\boldsymbol{f}_{\boldsymbol{\mu}}$ in Eq. (10) represent the inviscid convective fluxes and the diffusive fluxes, respectively. The convective fluxes $\boldsymbol{f}$ defined in terms of the relative velocity $\boldsymbol{u}-\boldsymbol{u}_{\boldsymbol{b}}$ is

$$
\boldsymbol{f}=\left[\begin{array}{ccc}
\rho\left(u-u_{b}\right) & \rho\left(v-v_{b}\right) & \rho\left(w-w_{b}\right) \\
\rho u\left(u-u_{b}\right)+p & \rho u\left(v-v_{b}\right) & \rho u\left(w-w_{b}\right) \\
\rho v\left(u-u_{b}\right) & \rho v\left(v-v_{b}\right)+p & \rho v\left(w-w_{b}\right) \\
\rho w\left(u-u_{b}\right) & \rho w\left(v-v_{b}\right) & \rho w\left(w-w_{b}\right)+p \\
\rho\left(E+\frac{p}{\rho}\right)\left(u-u_{b}\right) & \rho\left(E+\frac{p}{\rho}\right)\left(v-v_{b}\right) & \rho\left(E+\frac{p}{\rho}\right)\left(w-w_{b}\right)
\end{array}\right]
$$

where $\boldsymbol{u}_{\boldsymbol{b}}=\left(u_{b}, v_{b}, w_{b}\right)^{\boldsymbol{T}}$ is the grid velocity vector. The diffusive fluxes due to the viscous shear stresses, thermal diffusion and turbulence diffusion can be written as 


$$
\boldsymbol{f}_{\boldsymbol{\mu}}=\left[\begin{array}{ccc}
0 & 0 & 0 \\
\tau_{x x} & \tau_{x y} & \tau_{x z} \\
\tau_{y x} & \tau_{y y} & \tau_{y z} \\
\tau_{z x} & \tau_{z y} & \tau_{z z} \\
u \tau_{x x}+v \tau_{x y}+w \tau_{x z}-q_{x} & u \tau_{y x}+v \tau_{y y}+w \tau_{y z}-q_{y}+v \tau_{z y}+w \tau_{z z}-q_{z}
\end{array}\right]
$$

where $\tau_{\alpha \beta}$ with $\alpha, \beta \in\{x, y, z\}$ is the stress tensor expressed as

$$
\tau_{\alpha \beta}=\mu\left(\partial_{\alpha} u_{\beta}+\partial_{\beta} u_{\alpha}\right)-\frac{2}{3} \mu \delta_{\alpha \beta} \partial_{\alpha} u_{\beta},
$$

and

$$
q_{\alpha}=-k \partial_{\alpha} \Theta
$$

In the above equations, $\mu$ represents the dynamic viscosity, $k$ the thermal conductivity, and $\Theta$ the temperature. The stress tensor $\tau_{\alpha \beta}$ is the general form of the shear stresses in $\boldsymbol{f}_{\mu}$.

The fluid governing equations can be discretized into the following semi-discrete form

$$
\frac{d w}{d t}+R(w)=0 .
$$

By applying the dual-time method with a second-order implicit scheme, Eq. (17) could be reformulated into the following forms:

$$
\frac{d w^{n+1}}{d t^{*}}+R^{*}\left(w^{n+1}\right)=0,
$$

and

$$
R^{*}\left(w^{n+1}\right)=\frac{3 w^{n+1}-4 w^{n}+w^{n-1}}{2 \Delta t}+R\left(w^{n+1}\right) .
$$

The spatial discretization in the above equations is done based on a finite volume approach. The cell-centred based method is used to calculate gradients. The discretization of the convective fluxes is based on a secondorder Roe's flux-difference splitting scheme for Navier-Stokes equations. The discretization of the diffusive fluxes is based on a second-order artificial or matrix dissipation scheme. To cope with the complicated moving mesh cases, a structured multi-block mesh is generated ${ }^{1}$.

\section{Structural dynamics}

The structural elastic equations could be discretized in a finite-element model as follows:

$$
M \ddot{x}_{s}+C \dot{x}_{s}+K x_{s}=F,
$$

where $\boldsymbol{M}$ denotes the mass matrix, $\boldsymbol{C}$ represents the structural damping matrix, $\boldsymbol{K}$ is the stiffness matrix and $\boldsymbol{F}$ is the fluid dynamic force acting on the object surface. 
By finding the mode shape $\widetilde{\boldsymbol{\Phi}}$, a relationship equation could be defined as

$$
x_{s}=\widetilde{\boldsymbol{\Phi}} \boldsymbol{q},
$$

where $\boldsymbol{q}$ is the vector of the principal coordinates. Eq. (20) can be premultiplied by the transpose mode shape $\widetilde{\boldsymbol{\Phi}}^{T}$ as:

$$
\widetilde{\boldsymbol{\Phi}}^{T} \boldsymbol{M} \widetilde{\boldsymbol{\Phi}} \ddot{\boldsymbol{q}}+\widetilde{\boldsymbol{\Phi}}^{T} \boldsymbol{C} \widetilde{\boldsymbol{\Phi}} \dot{\boldsymbol{q}}+\widetilde{\boldsymbol{\Phi}}^{T} \boldsymbol{K} \widetilde{\boldsymbol{\Phi}} \boldsymbol{q}=\widetilde{\boldsymbol{\Phi}}^{T} \boldsymbol{F}
$$

By assuming linear combination between damping matrix and mass and stiffness matrices, Eq. (22) and Eq. (21) could be decoupled and the $j$ th equations are shown below:

$$
\ddot{q}_{j}+2 \zeta_{j} \omega_{j} \dot{q}_{j}+\omega_{j}^{2} q_{j}=\frac{\widetilde{\phi}_{j}}{m_{j}} \boldsymbol{F}
$$

and

$$
\Delta \boldsymbol{x}_{\boldsymbol{s}}=\sum_{j=1}^{N} q_{j} \tilde{\phi}_{j}
$$

where $\zeta_{j}$ is the modal damping ratio, $\omega_{j}$ is the natural frequency, and $m_{j}$ is the mass matrix in the $j$ th diagonal element. $\tilde{\phi}_{j}^{T}$ is the $j$ th element of mode shape $\widetilde{\boldsymbol{\Phi}}^{T}$ defined as

$$
\widetilde{\boldsymbol{\Phi}}^{T}=\left[\tilde{\phi}_{1}, \tilde{\phi}_{2}, \tilde{\phi}_{3}, \ldots, \tilde{\phi}_{N}\right]^{T}
$$

Following Alonso and Jameson ${ }^{25}$, the first-order differential equations are defined as

$$
\begin{gathered}
x_{1 j}=q_{j}, \\
\dot{x}_{1 j}=x_{2 j},
\end{gathered}
$$

and

$$
\dot{x}_{2 j}=\frac{\widetilde{\phi}_{j}^{T}}{m_{j}} \boldsymbol{F}-2 \zeta_{j} \omega_{j} x_{2 j}-\omega_{j}^{2} x_{1 j}
$$

Thus, the model equation could be rewritten as

$$
\dot{\boldsymbol{X}}_{\boldsymbol{j}}=\boldsymbol{A}_{\boldsymbol{j}} \boldsymbol{X}_{\boldsymbol{j}}+\boldsymbol{F}_{\boldsymbol{j}}, \quad j=1 \ldots N
$$

where,

$$
\begin{gathered}
\dot{\boldsymbol{X}}_{\boldsymbol{j}}=\left[\begin{array}{l}
\dot{x}_{1 j} \\
\dot{x}_{2 j}
\end{array}\right], \\
\boldsymbol{A}_{\boldsymbol{j}}=\left[\begin{array}{cc}
0 & 1 \\
-\omega_{j}^{2} & -2 \zeta_{j} \omega_{j}
\end{array}\right],
\end{gathered}
$$


and

$$
\boldsymbol{F}_{j}=\left[\begin{array}{c}
0 \\
\tilde{\phi}_{j}^{T} \\
\frac{m_{j}}{F}
\end{array}\right] .
$$

Then, the model equation can be further discretized by the pseudo-time $t^{*}$ as

$$
\frac{d z_{j}^{n+1}}{d t^{*}}+R_{s}^{*}\left(z_{j}^{n+1}\right)=0,
$$

and

$$
R_{s}^{*}\left(z_{j}^{n+1}\right)=\frac{3 z_{j}^{n+1}-4 z_{j}^{n}+z_{j}^{n-1}}{2 \Delta t}+R_{s}\left(z_{j}^{n+1}\right)
$$

where

$$
z_{j}=\left[\begin{array}{l}
z_{1 j} \\
z_{2 j}
\end{array}\right]
$$

and

$$
\frac{d z_{(1,2) j}}{d t^{*}}=\omega_{j}\left(-\zeta_{j} \pm \sqrt{\zeta_{j}^{2}-1}\right) z_{(1,2) j}+\frac{\sqrt{\zeta_{j}^{2}-1} \mp \zeta_{j}}{2 \sqrt{\zeta_{j}^{2}-1}} \frac{\widetilde{\phi}_{j}^{T}}{m_{j}} F
$$

In the current in-house code, Eq. (33) is coupled with Eq. (18). Specifically, the foil displacement data are employed by the fluid residual calculation as the flow boundary condition and the fluid parameters employed by the structure residual calculation as the hydrodynamic forcing. The detailed fluid-structure coupling algorithm is provided in the following.

\section{Fluid-structure coupling}

Since the fluid domain and the structure domain are calculated independently during the iterations, it is necessary to pass the fluid load from the fluid solver to the structure solver to calculate the structure deformation, and pass the structure displacement from the structure solver to the fluid solver as the boundary conditions to calculate the fluid forces. A linear transformation equation between the fluid domain and the structure domain is used to exchange information between them. The displacement $\Delta x_{f}$ of the fluid dynamic grid is expressed in terms of the structural grid displacements $\Delta x_{s}$ using a transformation matrix $\boldsymbol{S}$ as

$$
\Delta x_{f}=S \Delta x_{s}
$$

Since the motion of every node needs to meet the requirements of conservation of work, both fluid mesh nodes and structure mesh nodes have to obey the following equations: 


$$
\boldsymbol{f}_{s}^{T} \Delta \boldsymbol{x}_{\boldsymbol{s}}=\boldsymbol{f}_{f}^{T} \Delta \boldsymbol{x}_{\boldsymbol{f}}
$$

Eq. (37) and Eq. (38) lead to

$$
\Delta \boldsymbol{f}_{s}=\boldsymbol{S}^{T} \Delta \boldsymbol{f}_{f}
$$

In the transformation equations above, $\boldsymbol{f}_{\boldsymbol{s}}$ represents the force vector acting on the structure nodes and $\boldsymbol{f}_{\boldsymbol{f}}$ represents the force vector acting on the fluid nodes.

In order to find a proper transformation matrix $\boldsymbol{S}$, the Constant-Volume Tetrahedron (CVT) method is used. Since the volume of each associated tetrahedron should be a non-zero value and kept as a constant during the structural deformation, the CVT method can only be adopted to three-dimensional FSI modelling. Therefore, all two-dimensional cases in the present study are simulated by using a three-dimensional model. The details of this modelling strategy are described in the following.

\section{Numerical issues}

The computational domain and mesh for both the fluid and the structure are shown in Fig. 3. The model size in the spanwise direction is one chord length. As suggested in the literature ${ }^{26,27,28}$, applying Symmetry Boundary Condition (SBC) at the tips of the foil can effectively mitigate the three-dimensional effects and achieve two-dimensional simulation. It is therefore used in the following two-dimensional simulations.

The structure nodes are applied as an in-line mesh along the foil centreline shown in the lower left corner of Fig. 3 (a). An example of the CVT association between fluid node and structure nodes is displayed in the top right corner of Fig. 3 (a). A uniform constant velocity in the $\mathrm{x}$ direction is applied as the incoming flow for far field boundary condition for both inlet and outlet boundaries. The foil is set to be 50c (c is the foil chord length) away from the incoming flow and outflow boundaries. The two-dimensional view for both fluid mesh and structure mesh are shown in Fig. 3 (b) and (c), respectively. The grid resolution test (as shown in Fig. 4) and time-step size verification test (as shown in Table 1) are carried out for present study. Medium mesh and $\Delta t=T / 64$ with 300 iterations per time step are selected for present study to achieve a good calculation accuracy with an acceptable calculation time.

\subsection{Validations}

The numerical algorithms applied herein have been extensively validated in our previous publications (see Ref. 1, 29 and 30). In the current study, further investigations on a three-dimensional plunging-wing propeller have been conducted. The results predicted by our numerical model at $R e$ of 20000 are compared with the results by Heathcote et al. ${ }^{21}$ and Young ${ }^{31}$ in Fig. 5, where the non-dimensional Garrick frequency $\left(K_{G}\right)$ is defined 
as $K_{G}=\pi f_{0} c / U_{\infty}$. Both three-dimensional and two-dimensional (whose mesh have the same topological structure as that in XY cross-section of the three-dimensional model) simulations by using our in-house code have been compared with these results. Good agreement is shown in all cases.

In order to test the accuracy of our model in predicting flow energy harvesting, we examine the dynamics and energy harvesting performance of a pitching and heaving wing. In that case, the plunging amplitude is the same as the chord length. The foil pitches at 1/3 chordlength from its leading edge and the pitch amplitude is $75^{\circ}$. The phase angle between heave and pitch is $\pi / 2$. The time histories of drag coefficient, lift coefficient and power coefficient at a reduced frequency of 0.14 are shown in Fig. 6 (a)-(c) and the dependence of the timeaveraged energy extraction efficiency upon the reduced frequency is shown in Fig. 6 (d). They compare very well with the published results of Kinsey and Dumas ${ }^{18,33}$. It is not exactly the same for these instantaneous results as well as the time averaged results at high flapping frequency region. It is because the numerical algorithms of the fluid solvers used in these two studies are different (Kinsey and Dumas ${ }^{18,33}$ use commercial software FLUENT and present study use in-house code). In addition, the turbulence model used in present study is also different from them (S-A model was used by Kinsey and Dumas ${ }^{18,33}$ while present study use $k$ - $w$ model) which cause some different on the numerical results. Even though, the results from Kinsey and Dumas ${ }^{18,33}$ and present calculation follow the same trend which shows the dependence of present solver.

To validate the capability of this code in solving fluid-structure coupling problems, additional simulation is performed for a three-dimensional flapping wing with spanwise flexibility and compared with the experimental results by Heathcote et al. ${ }^{21}$ and numerical results by Gordnier et al. ${ }^{22,24}$. Two different types of flexible material, i.e. effective stiffness of $2.14 \times 10^{2}$ and $4.07 \times 10^{1}$, are studied and the instantaneous plots of some predicted parameters and flow fields are compared with Heathcote et al. ${ }^{21}$ and Gordnier et al. ${ }^{22}$. The problem addressed is a three-dimensional NACA0012 rectangular wing with a heaving motion in water at $\operatorname{Re}$ of $3 \times 10^{4}$. The chord length is $0.1 \mathrm{~m}$ and the span length is $0.3 \mathrm{~m}$. The plunging amplitude is $1.75 \times 10^{-2} \mathrm{~m}$ with the oscillating frequency of $1.74 \mathrm{~Hz}$. To construct the chordwise flexibility, a thin stiffener along the centreline of foil is used, which is covered with a PDMS rubber. The thickness of the stiffener is $10^{-2}$ chord length. In the foil with effective stiffness of $2.14 \times 10^{2}$, the material properties of the stiffener are chosen so that the Young's modulus of $210 \mathrm{GPa}$ and density ratio of 7.8 . The instantaneous thrust, foil tip displacement and lift coefficient are shown in Fig. 7 (a)-(c). The vorticity contours at two instantaneous time of $t / T=0$ and $t / T=0.25$ are compared in Fig. 8 with the experimental results. In the wing with effective stiffness of $4.07 \times 10^{1}$, the Young's modulus is set as 70GPa and the density ratio as $2.7^{24}$. Since present Fluid Structure Interaction code using a linear structure 
solver with linear coupling strategy between fluid solver and structure solver, the non-linear structure effect will be ignored during the calculation. Blade tip effect of the three-dimensional flapping wing with spanwise flexibility case is also ignored during the calculation. Corresponding results are presented in Fig. 9. All of the above results prove the accuracy of our code to cope with fluid-structure interaction problems.

\section{Results}

We start our investigation by using real metal materials for the stiffener. Specifically, two types of material are used, Copper $(\mathrm{Cu})$ and Tungsten Carbide (WC). This is because copper is a common metal with relatively high flexibility. Tungsten Carbide, on the other hand, possesses large Young's modulus coefficient and density. The detailed parameters used in our study are listed in Table 2. Numerical simulations are carried out for the above two stiffener materials and the results are compared with those with a rigid foil. Two pitching amplitudes, $60^{\circ}$ and $75^{\circ}$, are applied. These are close to the pitching amplitudes with high efficiency energy harvesting (see e.g. Ref. 15). The effective stiffness are $5.25 \times 10^{1}$ and $2.62 \times 10^{2}$ for Copper and Tungsten Carbide stiffener, respectively.

\subsection{Energy harvesting performance with stiffeners of real materials}

As shown in Eq. (5), the key parameter to quantify the energy extraction performance of an oscillating foil device is the efficiency $\eta$. In Fig. 10 (a) we compare the energy extraction efficiency of the copper stiffener foil (hereafter referred to as $\mathrm{Cu}$ ), the tungsten carbide stiffener foil (referred to as WC) and the rigid foil at different reduced frequencies. Based on these results, flexible foils show certain level of improved performance as compared to their rigid counterparts. For example, the peak efficiency of WC reaches $20 \%$ at the pitching angle of $60^{\circ}$ and $43 \%$ at the pitching angle of $75^{\circ}$, while the corresponding results for the rigid foil are $19 \%$ and $35 \%$, respectively. $\mathrm{Cu}$ also reaches $36 \%$ at $60^{\circ}$ pitching angle. The efficiency enhancement of $\mathrm{Cu}$ (compared with the rigid foil) is $17.4 \%$ at a reduced frequency of 0.15 and $32.2 \%$ with $f^{*}=0.25$. Since the structural model used herein is linear, which is not accurate in simulating large deformation, it is not used to study cases with low stiffness at high pitching angle.

Within the large reduced frequency region $\left(f^{*}=0.18-0.25\right)$, where rigid foils usually present a decayed performance or even degrade to negative power extraction, both $\mathrm{Cu}$ and $\mathrm{WC}$ flexible foils still exhibit a positive energy extraction with the efficiency much higher than that of the rigid foil.

The trailing edge displacement amplitude $\beta_{0 \_a m p}$ shows different magnitudes and variation trends for the two flexible foils as displayed in Fig. 10 (b). Because of the small Young's modulus coefficient for $\mathrm{Cu}$, the 
trailing edge displacement of $\mathrm{Cu}$ is larger than that of $\mathrm{WC}$. For $\mathrm{Cu}$, the maximum value of such displacement is around 0.063 .

Apart from the time-averaged results, the instantaneous displacement and force results at a reduced frequency of 0.18 are picked up for a detailed analysis.

The instantaneous trailing edge displacement for the two flexible foils and the rigid foil are plotted in Fig. 11 (a). It is clear that the peak value of 0.055 is reached at $t / T=3 / 8$ for the $\mathrm{Cu}$ foil. Large trailing edge displacement is achieved during $t / T=2 / 8$ and $3 / 8$ for the WC foil.

Fig. 11 (b) shows the lift coefficient along with the foil heaving velocity $(d h / d t)$. It is observed that the structural flexibility significantly increases the peak lift coefficient (as shown in the curve of $\mathrm{Cu}$ ). In addition, within the timeframe from $t / T=2 / 8$ to $4 / 8$, the flexible foils achieve higher $C_{L}$ than the rigid foil. As concluded by Xiao et al. ${ }^{30}$, it has a positive contribution to the time-mean efficiency over one cycle if the force or moment has the same sign as the heaving or pitching velocity. Therefore, an increased $C_{L}$ in the above mentioned time frames contribute positively to the overall efficiency.

The variations of moment coefficient $(M z)$ and the pitch velocity $(d \theta / d t)$ are presented in Fig.11 (c). A phase lag of $180^{\circ}$ for $\mathrm{Mz}$ and $d \theta / d t$ is shown so that the pitching motion contributes negatively to the overall energy extraction. The flexibility of foil leads to a slightly decrease in $M z$ during $t / T=0 / 8$ and $3 / 8$, reduces the power expenditure in pitching so that the overall energy harvesting performance is improved.

The instantaneous power coefficient is shown in Fig. 11 (d). It is clear that, with flexible foils, the positive instantaneous power is enhanced while the negative power is reduced.

Fig. 12 shows the vorticity contour for $\mathrm{Cu}, \mathrm{WC}$, and the rigid foil at $f^{*}=0.18$ and pitch amplitude of $60^{\circ}$ within half an oscillating period. Due to the small trailing edge deformation of the flexible foil, it is difficult to illustrate it in the original contour plot. Thus, schematic representations with five times scale of the deformation have plotted to help the visualization. The most pronounced characteristic of the flexible foils compared to the rigid foil is the enhanced leading edge vortices. This is explained by the synchronization between the development of LEV and the foil deformation. As demonstrated in Fig. 12, at $t / T=0$ the centreline of the foil is almost straight and the vorticity generated from the leading edge is attached to the upper surface of the foil. At $t / T=1 / 8$ the hinder part of the foil starts bending downwards, making it hard for the leading edge vorticity to remain attached. This encourages the separation of the leading edge vorticity and the growth of the leading edge vortex. As indicated in $\mathrm{Zhu}^{34}$, the strength of leading edge vortices is closely associated with the energy harvesting performance. Enhanced leading edge separation and well-formed leading edge vortices usually lead 
to high efficiency (see Figs. 7 and 8 in Ref. 34). As can be observed from Fig. 11 (b) and (c), the phase difference between the flexible blade and rigid blade in terms of lift and moment coefficients are not as remarkable as that of the amplitude difference. It can also confirmed by the contour plot of Fig. 12. Thus the flexible effect has few influences on the timing of LEV. Based on this, a possible mechanism of performance improvement is the enhanced leading edge separation due to the structural deformations of the foil. The optimal $f^{*}$ is also increased due to this effect. When studying the flexible wing or membrane problems, previous researchers ${ }^{21,22,23}$ often used real materials with specific combinations of material properties (e.g. Young's modulus and density) as we did herein. On the other hand, several studies ${ }^{8,23}$ suggest that it is more interesting to decouple the Young's modulus coefficient and the density ratio and examine their effects independently. Hereby, we will study the effects of these two parameters separately by constructing some virtual materials with (a) a constant density ratio but variable Young's modulus coefficient and (b) a constant Young's modulus coefficient with different density ratios. In these cases, the foil oscillation reduced frequency is fixed as 0.18 and the pitching amplitude is set as $60^{\circ}$.

\subsection{Young's modulus coefficient effect}

In this part, six different Young's modulus coefficients ranging from $8.15 \times 10^{7} \mathrm{~Pa}$ to $1.02 \times 10^{9} \mathrm{~Pa}$ are investigated (further reduction of the Young's modulus coefficient leads to large foil deformations that are beyond the capacity of the linear structural model). The density ratio is set as 8.9. The effective stiffness for these cases varies from $3.82 \times 10^{1}$ to $4.77 \times 10^{2}$, accordingly. The detailed parameters are summarized in Table 3 .

Fig. 13 shows the variation of the energy extraction efficiency with Young's modulus coefficient. With the increase of Young's modulus coefficient, the efficiency decreases and eventually approaches the rigid foil result. An empirical formulation is generated to quantify this curve and expressed as:

$$
\eta=a_{1} E^{2}+a_{2} E+a_{3},\left(8 \times 10^{10} P a \leq E \leq 1 \times 10^{12} P a\right) .
$$

where $a_{1}=3 \times 10^{-25} \mathrm{~Pa}^{-2}, a_{2}=-5 \times 10^{-13} \mathrm{~Pa}^{-1}$ and $a_{3}=0.3692 \mathrm{~Pa}^{0}$.

The peak trailing edge displacement at different values of Young's modulus coefficient is shown in Fig. 14 (a). Similar to the efficiency plot in Fig. 13, the displacement reduces rapidly with the increase of Young's modulus coefficient. This is well reflected by the profoundly smaller $\beta_{0}$ with larger $E_{c}$ in Fig. 14 (b), where the instantaneous trailing displacement is shown within two oscillating periods.

The instantaneous lift coefficient $\left(C_{L}\right)$, moment coefficient $(M z)$ and power coefficients $\left(C_{o p}\right)$ are analyzed in Fig. 15. With the increase of Young's modulus coefficient, a drop of lift coefficient and moment coefficient can 
be seen in Fig. 15 (a) and (b). This leads to the drop of the instantaneous power Fig. 15 (c). It agrees well with the tendency of the time-mean energy efficiency as seen in Fig. 13.

\subsection{Density ratio effect}

In this part, nine density ratios ranging from 7 to 1500 are investigated with a fixed Young's modulus coefficient of $1.12 \times 10^{8} \mathrm{~Pa}$. The detailed parameters are listed in Table 4 .

Fig. 16 shows the energy harvesting efficiency at various density ratios in a logarithmic graph. With the increase of the density ratio, the efficiency is dramatically decreased. As the density ratio is larger than 700 , the foil switches from an energy extraction state (positive $\eta$ ) to an energy consumption state (negative $\eta$ ).

The variation of trailing edge displacement with density ratio is shown in Fig. 17 (a). The maximum displacement occurs at $D R=700$ with the magnitude of 0.239 , which actually is quite large compared with the pitching angle of $60^{\circ}$.

In Fig. 17 (b) and (c) the instantaneous trailing edge displacement with small density ratios $(D R=8.9$ and 30$)$ and large $D R \mathrm{~s}(D R=700,1200$ and rigid) are presented. Apart from the trend of peak beta variation with $D R \mathrm{~s}$, which is already shown in Fig. 17 (a), it is also noted that when the density ratio is near 700, the instantaneous displacement profile presents a very regular sinusoid containing one dominant frequency. It is determined from our results that the phase difference between trailing edge displacement and corresponding heave for small density ratios $(D R<700)$ is around $180^{\circ}$. However, when the density ratio is increased to 700 , the phase lag becomes $90^{\circ}$. For $D R=1200$, this angle changes to $0^{\circ}$. This phase variation is consistent with the response of an oscillator around its natural frequency. It is also found that both foil inertia and fluid forces are influenced for the foil deformation and the fluid forces play a leading role on the foil deformation for present cases. This is coinciding with $\mathrm{Zhu}^{8}$ which defined as fluid driven condition. When the foil natural frequency approach to the foil oscillating frequency by varying the $D R$, the effect from foil inertia is observed increased significantly. When the foil natural frequency far away from the oscillating frequency, it is found that the contribution from the foil inertia to the deformation is limited.

Fig. 18 (a) - (c) show the density ratio effect on the instantaneous lift, moment and power coefficients associated with low-density ratios $(D R=8.9$ and 30$)$. It is clear that the difference of various density ratios mostly affects the peak lift coefficient, particularly within the time frames of $t / T=2 / 8$ to $7 / 16$. In Fig. 19 (a)-(c), the instantaneous plots are shown at large density ratios $(D R=700$ and 1200). A large fluctuation occurs at $D R=700$, which is under a resonance condition. 
From the vorticity plots in Fig. 20, it is seen that cases with smaller density ratio are characterized by enhanced development of LEV. An increased LEV generation, according to our discussion in Sec. 3.1, is beneficial to energy extraction. Moreover, within the time slot between $t / T=2 / 8$ and $3 / 8$, the LEV re-attaches to the upper surface of the foil near the trailing edge, creating a counter-clockwise moment. It coincides with the pitching motion of the foil at that instant so that it provides additional contribution to the energy harvesting. This beneficial effect is not achieved in cases with higher density ratio. In contrast, at these cases (e.g. $D R=700$ or 1200) during the counter-clockwise pitching motion of the foil there is significant vorticity attached to the lower surface of the foil near the trailing edge. The associated low pressure induces a clockwise moment so that there is negative contribution to energy harvesting.

\section{Conclusions and Discussions}

Using a fully-coupled Fluid-Structure-Interaction (FSI) algorithm, we numerically investigated the effect of structural flexibility upon the energy extraction capacity of an oscillating foil with realistic internal structure characterized by a stiffener near the trailing edge. The power generation predicted by our model is actually the net energy flux from the flow field to the foil. In a real system (such as the one by Kinsey and Dumas ${ }^{18,19}$ ), it is the extracted power minus the power input. For the stiffener, two types of real material (i.e. Copper and Tungsten Carbide) are tested with their different effective stiffness and density ratios. To distinguish the effects of Young's modulus coefficient and density ratio to the dynamic response and energy extraction efficiency, we also studied cases with stiffener made of virtual materials with arbitrary Young's modulus and density.

The simulation shows that the passive structural flexibility in the vicinity of trailing edge is capable of enhancing the overall energy extraction performance. Up to $32.2 \%$ increase in the efficiency is achieved by using a foil with copper stiffener. This is attributed to the enhancement of leading edge vortices associated with the synchronization between vorticity generation/shedding and foil deformation. Within the range of parameters we consider, both Young's modulus coefficient and density ratio affect the amplitude as well as the phase lag between the deformation (characterized by the instantaneous trailing edge displacement) and heave displacements. At sufficiently large density ratios, such change causes the oscillating foil to switch from energy extraction into energy consumption. A resonance phenomenon is also observed when the natural frequency of the foil coincides with its heaving frequency.

The beneficial effect of structural flexibility in the energy extraction of a flapping foil is reminiscent of the similar performance enhancement of flapping foils propellers using deformable structures (see for example Ref. 34, 35 and 36). There are, however, fundamental differences between these two phenomena. According to Katz 
and Weihs ${ }^{35}$, the key mechanism of the propulsion efficiency increase in a chord-wisely flexible foil is that the overall fluid dynamic force is redirected more towards the front (see Fig. 6 in Ref. 35). Another mechanism is that in flexible propellers the leading edge separation is suppressed due to the reduction in the effective angle of attack at the leading edge. In contrast, our current research indicates that structural flexibility in an energy harvester actually encourages the development of LEV, leading to higher capacity in energy extraction.

A leading edge flexibility study, where a flexible internal structure is installed in the leading edge area, has also been performed. This structural design, however, leads to reduced efficiency according to our results (this is similar to the conclusion by Tian et al. ${ }^{14}$, in which it is suggested that passive deformability at the leading edge is not beneficial to energy harvesting). Specifically, the passively induced instantaneous leading edge deformation is $90^{\circ}$ ahead of the foil heave displacement in phase. This leads to the decrease of the effective angle of attack at the leading edge and thus weakens the strength of LEV.

Lastly, in the present study the flexible foil is modeled in two dimensions and thus only the bending motion is considered. In reality, a three-dimensional flexible wing may present a combined bending and twist motion under the external forces. Therefore, performing a fully three-dimensional fluid-structure interaction analysis to account for foil passive torsion and bending will be our next task in the near future. In addition, since the investigation into this problem has just started and the fluid-structure interaction problem is not well understood, we believe that one needs to increase the complexity of the problem step by step. In particular, we notice that including some more components and part form the real device (such as electromechanical transducer which in general requires a semi-passive or a fully passive device) will introduce a large number of parameters so that it is hard to come up with a clean physical problem as we have. It is our belief that these parameters should be introduced in later studies when the physics of the basic system is well documented.

\section{Acknowledgments}

Results were obtained using the EPSRC funded ARCHIE-WeSt High Performance Computer (www.archiewest.ac.uk). EPSRC grant no. EP/K000586/1.

\section{References}

[1] Liu, W., Xiao, Q., \& Cheng, F. "A bio-inspired study on tidal energy extraction with flexible flapping wings." Bioinspiration \& biomimetics 8, no. 3 (2013): 036011.

[2] Xiao, Q., \& Zhu, Q. "A review on flow energy harvesters based on flapping foils." Journal of Fluids and Structures, 46 (2014): 174-191 
[3] McKinney, W., \& DeLaurier, J. "Wingmill: an oscillating-wing windmill." Journal of Energy 5, no. 2 (1981): 109-115.

[4] Young, J., Lai, J., \& Platzer, M. F. "A review of progress and challenges in flapping foil power generation." Progress in Aerospace Sciences 67 (2014): 2-28.

[5] Nakata, T., \& Liu, H. "Aerodynamic performance of a hovering hawkmoth with flexible wings: a computational approach." Proceedings of the Royal Society B: Biological Sciences (2012): 279(1729), 722-731.

[6] Combes, S. A., and T. L. Daniel. "Flexural stiffness in insect wings I. Scaling and the influence of wing venation." Journal of experimental biology 206, no. 17 (2003): 2979-2987.

[7] Combes, S. A., and T. L. Daniel. "Flexural stiffness in insect wings II. Spatial distribution and dynamic wing bending." Journal of Experimental Biology 206, no. 17 (2003): 2989-2997.

[8] Zhu, Q. "Numerical simulation of a flapping foil with chordwise or spanwise flexibility." AIAA journal 45, no. 10 (2007): $2448-2457$

[9] Shoele, K., \& Zhu, Q. "Leading edge strengthening and the propulsion performance of flexible ray fins." Journal of Fluid Mechanics 693 (2012): 402-432.

[10] Shoele, K. and Zhu, Q. "Performance of a wing with nonuniform flexibility in hovering flight." Physics of Fluids (1994present) 25, no. 4 (2013): 041901.

[11] Shyy, W., Aono, H., Chimakurthi, S. K., Trizila, P., Kang, C. K., Cesnik, C. E., \& Liu, H. "Recent progress in flapping wing aerodynamics and aeroelasticity." Progress in Aerospace Sciences 46, no. 7 (2010): 284-327.

[12] Daniel, T. L., \& Combes, S. A. "Flexible wings and fins: bending by inertial or fluid-dynamic forces?" Integrative and Comparative Biology 42, no. 5 (2002): 1044-1049.

[13] Yin, B. and Luo, H. "Effect of wing inertia on hovering performance of flexible flapping wings." Physics of Fluids (1994-present) 22, no. 11 (2010): 111902.

[14] Tian, F. B., Young, J., \& Lai, J. C. "Improving power-extraction efficiency of a flapping plate: From passive deformation to active control." Journal of Fluids and Structures 51 (2014): 384-392.

[15] Kinsey, T., \& Dumas, G. "Parametric study of an oscillating airfoil in a power-extraction regime." AIAA journal 46, no. 6 (2008): 1318-1330.

[16] Jones, K. D., and M. F. Platzer. "Numerical computation of flapping-wing propulsion and power extraction." AIAA paper 97 (1997): 0826.

[17] Jones, K. D., Lindsey, K., \& Platzer, M. F. "An investigation of the fluid-structure interaction in an oscillating-wing micro-hydropower generator." Advances in Fluid Mechanics, 2003, 36, 73-84.

[18] Kinsey, T., \& Dumas, G. "Computational fluid dynamics analysis of a hydrokinetic turbine based on oscillating hydrofoils." Journal of Fluids Engineering 134, no. 2 (2012): 021104.

[19] Kinsey, T., \& Dumas, G. "Optimal tandem configuration for oscillating-foils hydrokinetic turbine." Journal of fluids engineering 134, no. 3 (2012): 031103. 
[20] Heathcote, S., \& Gursul, I. "Flexible flapping airfoil propulsion at low Reynolds numbers." AIAA journal 45, no. 5 (2007): 1066-1079.

[21] Heathcote, S., Wang, Z., and Gursul, I., "Effect of spanwise flexibility on flapping wing propulsion." Journal of Fluids and Structures 24, no. 2 (2008): 183-199.

[22] Gordnier, R. E., Attar, P. J., Chimakurthi, S. K., \& Cesnik, C. E. "Implicit LES simulations of a flexible flapping wing." AIAA Paper 2960 (2010).

[23] Chimakurthi, S. K., Tang, J., Palacios, R., S. Cesnik, C. E., \& Shyy, W. "Computational aeroelasticity framework for analyzing flapping wing micro air vehicles." AIAA journal 47, no. 8 (2009): 1865-1878.

[24] Gordnier, R. E., Kumar Chimakurthi, S., Cesnik, C. E., \& Attar, P. J. "High-fidelity aeroelastic computations of a flapping wing with spanwise flexibility." Journal of Fluids and Structures 40 (2013): 86-104.

[25] Alonso J. J., and Jameson, A., "Fully-Implicit Time-Marching Aeroelastic Solutions", AIAA Paper 94-0056, Jan., 1994.

[26] Lifante, C., Frank, T., \& Kuntz, M. "Extension and Validation of the CFX Cavitation Model for Sheet and Tip Vortex Cavitation on Hydrofoils." In 5th Joint FZR \& ANSYS Workshop, Multiphase Flows: Simulation, Experiment and Application. 2007.

[27] Nicolato, P. C., \& Moreira, R. M. "Numerical Modeling of Water Wave Impact on Reservoirs." In Proceedings of the 20th International Congress of Mechanical Engineering. Gramado, pp. 1-9. 2009.

[28] Scott, D., M. Sabourin, S. Beaulieu, B. Papillon, and C. Ellis. "CFD model of an aerating hydrofoil." In IOP Conference Series: Earth and Environmental Science, vol. 22, no. 6, p. 062008. IOP Publishing, 2014.

[29] Xiao Q. and Liao W. "Numerical investigation of angle of attack profile on propulsion performance of an oscillating foil." Computers \& Fluids 39, no. 8 (2010): 1366-1380.

[30] Xiao, Q., Liao, W., Yang, S., \& Peng, Y. "How motion trajectory affects energy extraction performance of a biomimic energy generator with an oscillating foil?" Renewable Energy 37, no. 1 (2012): 61-75.

[31] Young, J. "Numerical simulation of the unsteady aerodynamics of flapping airfoils." PhD thesis, University of New South Wales, Australian Defence Force Academy, School of Aerospace, Civil and Mechanical Engineering., 2005.

[32] Garrick, I. E. "Propulsion of a flapping and oscillating airfoil." National Bureau of Standards, NACA-REPORT-567, 1936.

[33] Kinsey, T., \& Dumas, G. "Three-dimensional effects on an oscillating-foil hydrokinetic turbine." Journal of Fluids Engineering 134, no. 7 (2012): 071105.

[34] Zhu, Q. "Optimal frequency for flow energy harvesting of a flapping foil." Journal of fluid mechanics 675 (2011): 495 517.

[35] Katz, J., and D. Weihs. "Hydrodynamic propulsion by large amplitude oscillation of an airfoil with chordwise flexibility." Journal of Fluid Mechanics 88, no. 03 (1978): 485-497.

[36] Katz, J., \& Weihs, D. "Large amplitude unsteady motion of a flexible slender propulsor." Journal of Fluid Mechanics 90, no. 04 (1979): 713-723. 
Table 1 Time-step size verification of present study for a oscillating foil at $f^{*}=0.18$ and $\theta_{0}=60^{\circ}$

\begin{tabular}{ccc}
\hline \hline $\begin{array}{c}\text { number of time } \\
\text { steps per period }\end{array}$ & $\begin{array}{c}\text { Iterations } \\
\text { for one } \\
\text { time step }\end{array}$ & $\begin{array}{c}\text { Time } \\
\text { averaged } \\
C_{o p}\end{array}$ \\
\hline 128 & 400 & 0.433 \\
64 & 300 & 0.432 \\
32 & 200 & 0.406 \\
\hline \hline
\end{tabular}

Table 2 Case summary for real materials

\begin{tabular}{ccccccc}
\hline \hline Case & Material & $\begin{array}{c}\text { Young's modulus } \\
\text { coefficient }\end{array}$ & $\begin{array}{c}\text { Density ratio } \\
D R\end{array}$ & $\begin{array}{c}\text { Effective } \\
\text { stiffness } \Pi\end{array}$ & $\begin{array}{c}\text { Poisson } \\
\text { ratio }\end{array}$ & $\begin{array}{c}\text { Natural } \\
\text { frequency } \\
\text { ratio } F R\end{array}$ \\
\hline 1 & Copper $(\mathrm{Cu})$ & $1.12 \times 10^{8}$ & 8.9 & $5.25 \times 10^{1}$ & 0.34 & 7.55 \\
2 & $\begin{array}{c}\text { Tungsten } \\
\text { Carbide (WC) }\end{array}$ & $5.61 \times 10^{8}$ & 15.6 & $2.46 \times 10^{2}$ & 0.24 & 12.76 \\
\hline \hline
\end{tabular}

Table 3 Case summary for virtual materials used to test Young's modulus effect. The Poisson ratio $(v)$ for all cases is 0.34

\begin{tabular}{cccc}
\hline \hline Case & Young's modulus coefficient & Effective stiffness $\Pi$ & Natural frequency ratio \\
& & & 6.44 \\
\hline 1 & $8.15 \times 10^{7}$ & $3.82 \times 10^{1}$ & 7.55 \\
2 & $1.12 \times 10^{8}$ & $5.25 \times 10^{1}$ & 11.38 \\
3 & $2.55 \times 10^{8}$ & $1.19 \times 10^{2}$ & 16.88 \\
4 & $5.61 \times 10^{8}$ & $2.62 \times 10^{2}$ & 19.71 \\
5 & $7.65 \times 10^{8}$ & $3.58 \times 10^{2}$ & 22.75 \\
6 & $1.02 \times 10^{9}$ & $4.77 \times 10^{2}$ & \\
\hline \hline
\end{tabular}

Table 4 Case summary for virtual materials used to test density ratio effect. The effective stiffness $(I I)$ and Poisson ratio $(v)$ for all cases are $5.25 \times 10^{1}$ and 0.34 respectively

\begin{tabular}{ccc}
\hline Case & Density Ratio $D R$ & Natural frequency ratio $F R$ \\
\hline 1 & 7.0 & 8.54 \\
2 & 8.9 & 7.55 \\
3 & 12.0 & 6.53 \\
4 & 15.6 & 5.72 \\
5 & 20.0 & 5.05 \\
6 & 30.0 & 4.13 \\
7 & 70.0 & 2.70 \\
8 & 700.0 & 0.85 \\
9 & 1000.0 & 0.72 \\
10 & 1200.0 & 0.65 \\
11 & 1500.0 & 0.58 \\
\hline \hline
\end{tabular}




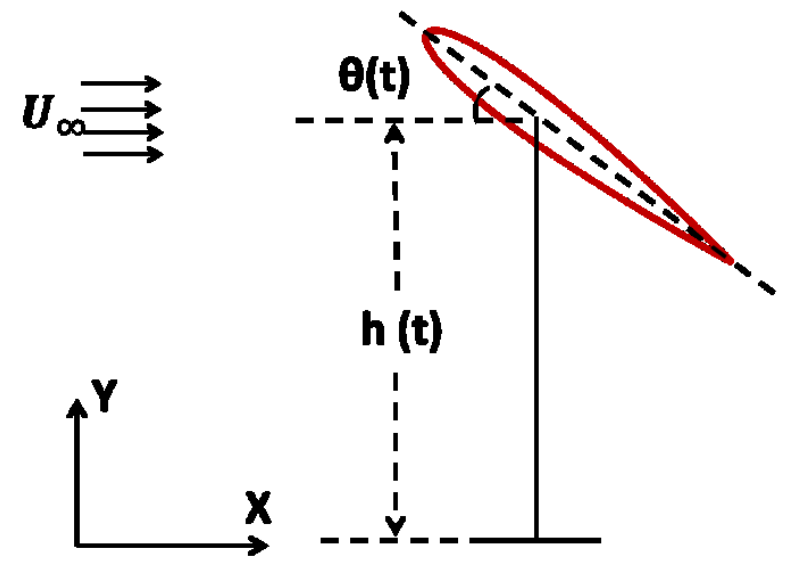

Figure 1 Schematic diagram for oscillating energy device

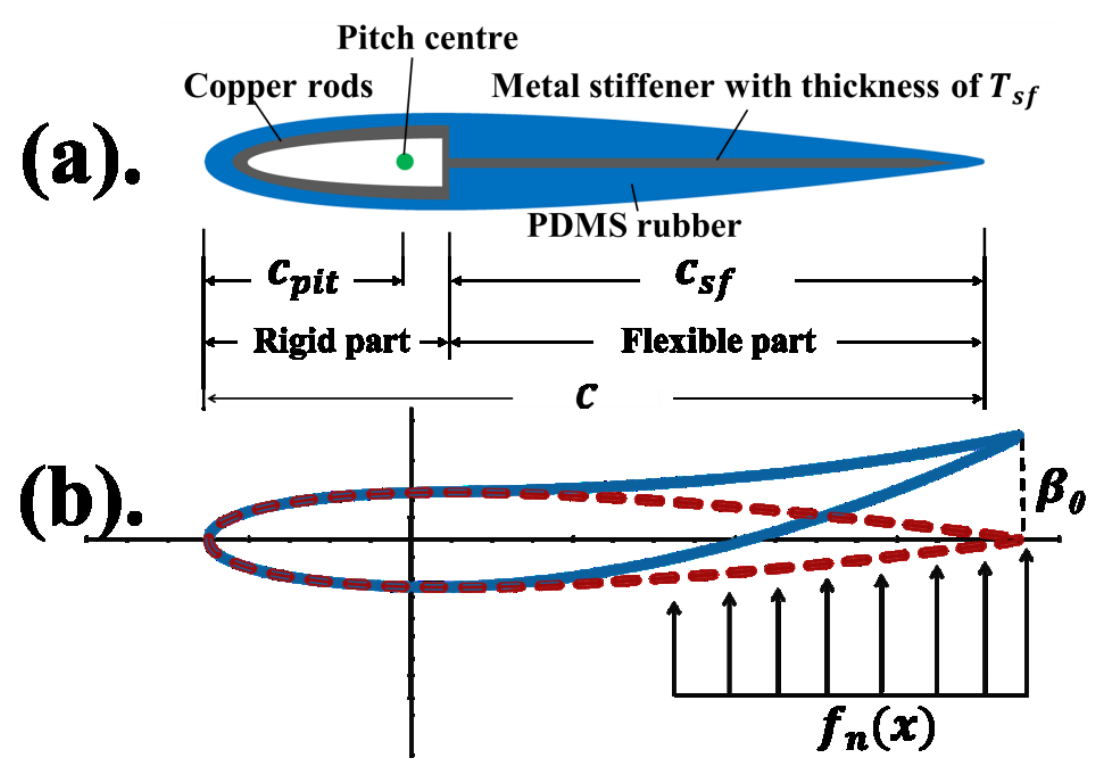

Figure 2 (a) Inner structural design for trailing edge flexible foil and (b) flexible foil displacement 


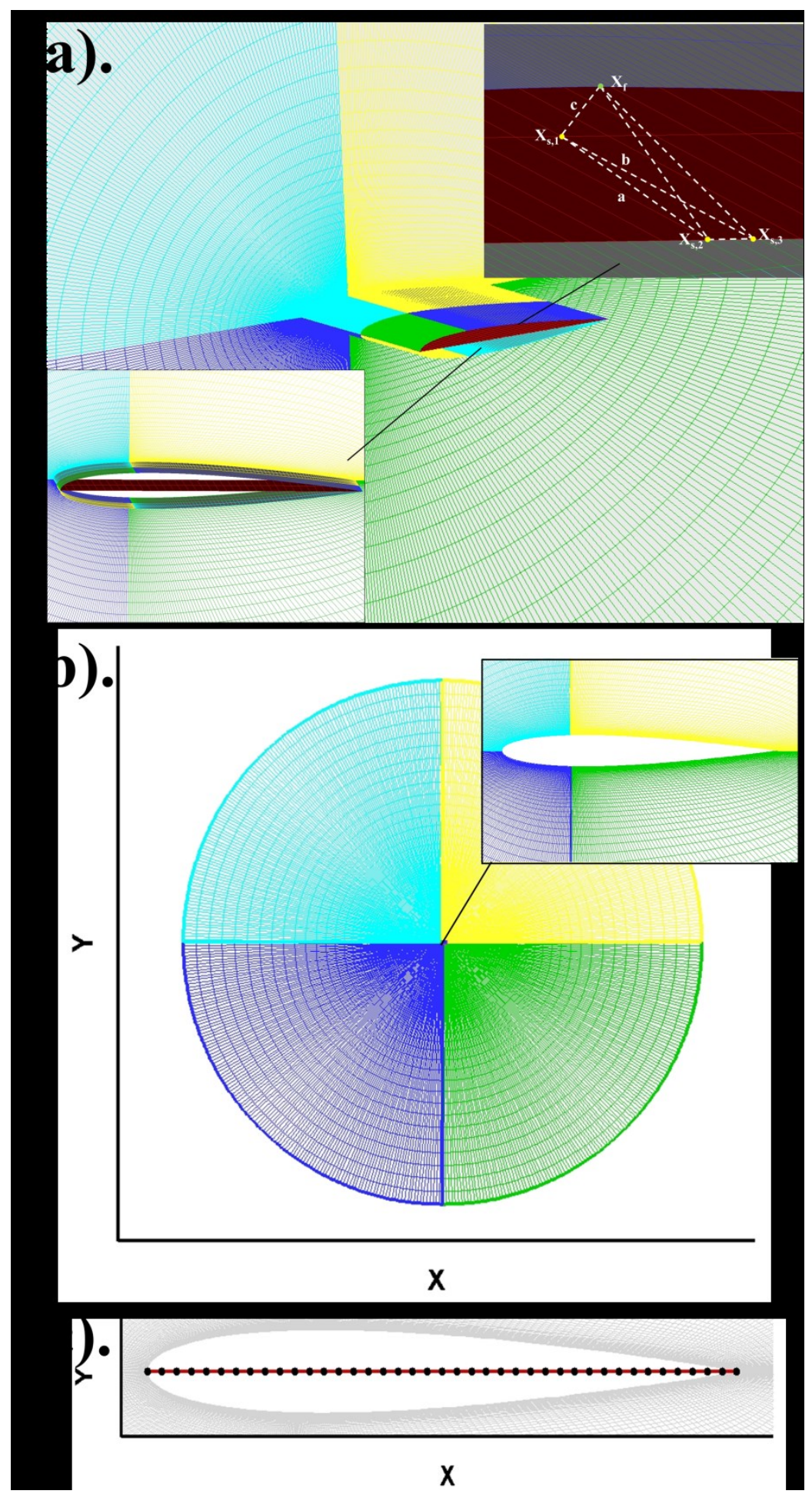

Figure 3 Computational domain: (a) 3D view for fluid mesh (cyan, yellow, blue and green represent different blocks) and structure mesh (red colour) and corresponding CVT mapping example; (b) Fluid mesh in 2D view; (c) Structure mesh in 2D view 


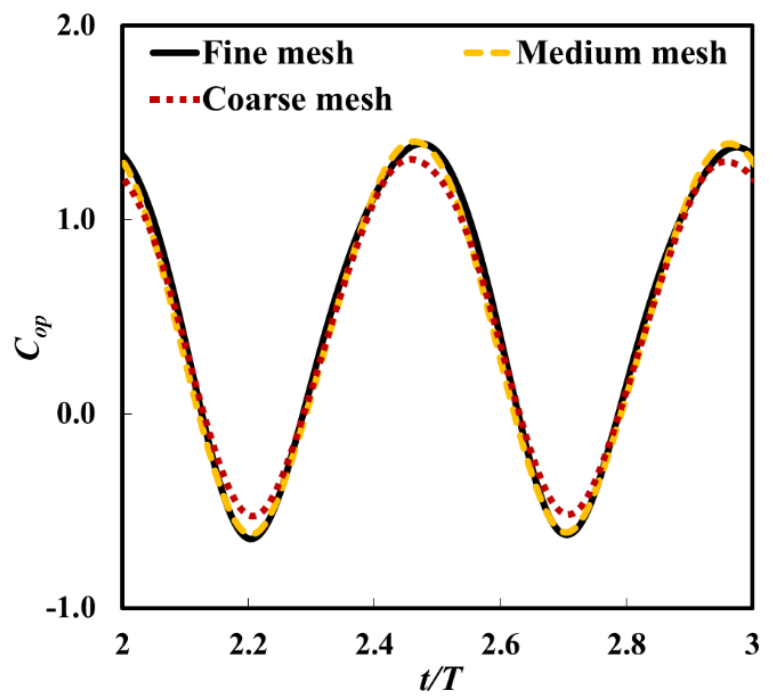

Figure 4. Mesh resolution test of present study for a oscillating foil at $f^{*}=0.18$ and $\theta_{0}=60^{\circ}$ 


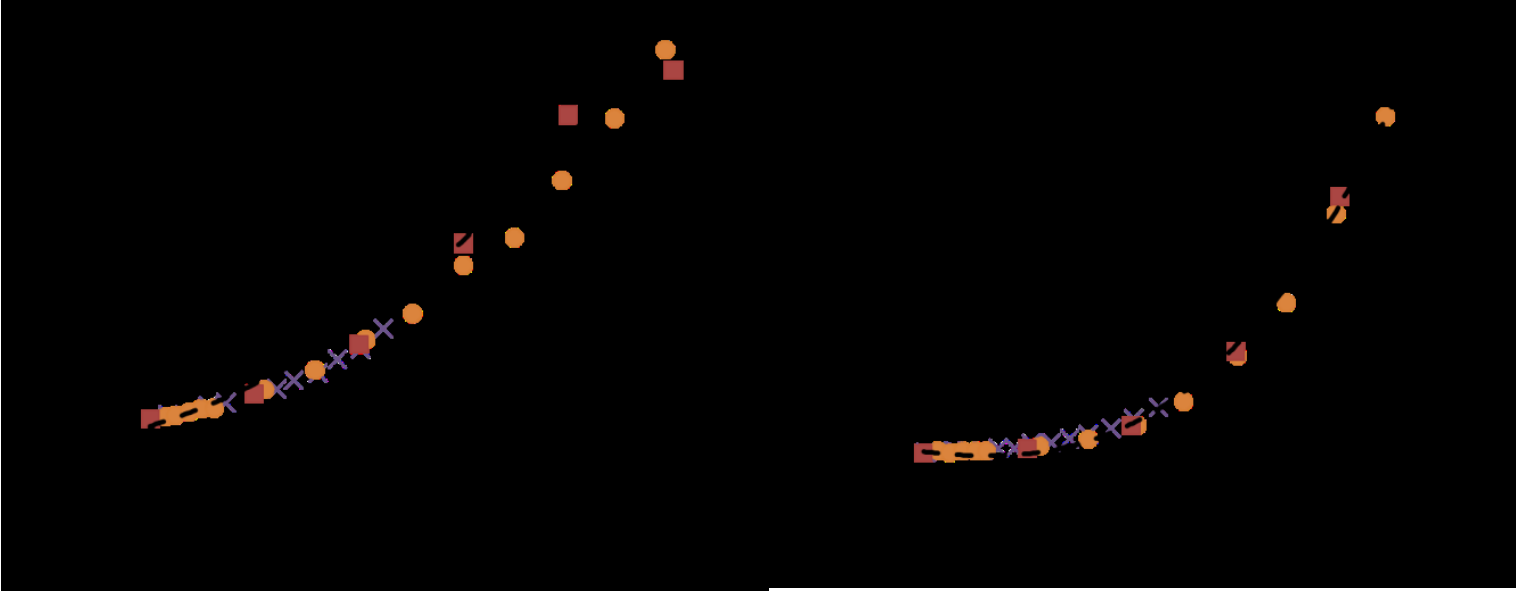

(a) Thrust coefficient

(b) power coefficient

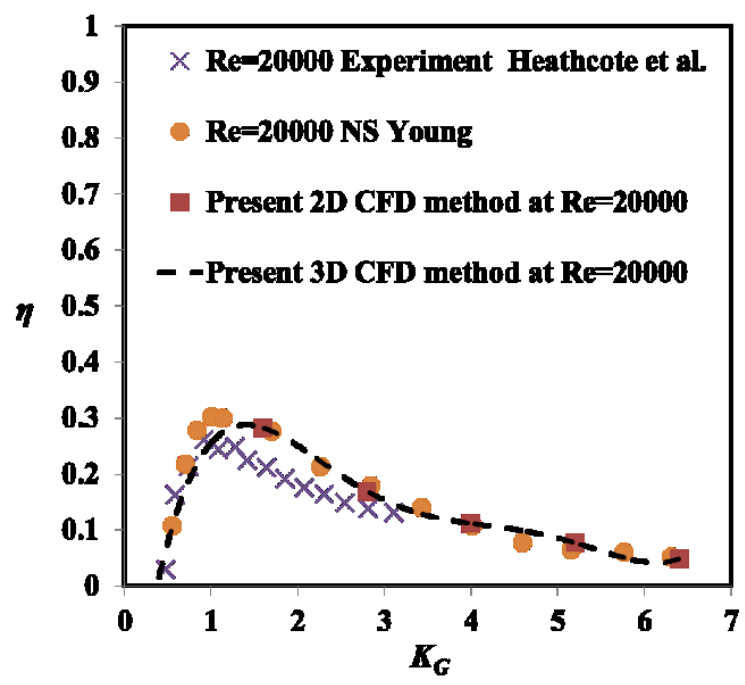

(c) efficiency

Figure 4 Validation results of a 3D rigid flapping foil with $A R=3.0$ withExperiment results at $R e=20000^{21}$, N-S numerical results at $R e=20000^{31}$, Present 2D CFD method at $R e=20000$ (achieved with a 3D method using SBC boundary conditions at the tips) and present 3D CFD method at $R e=20000$. 


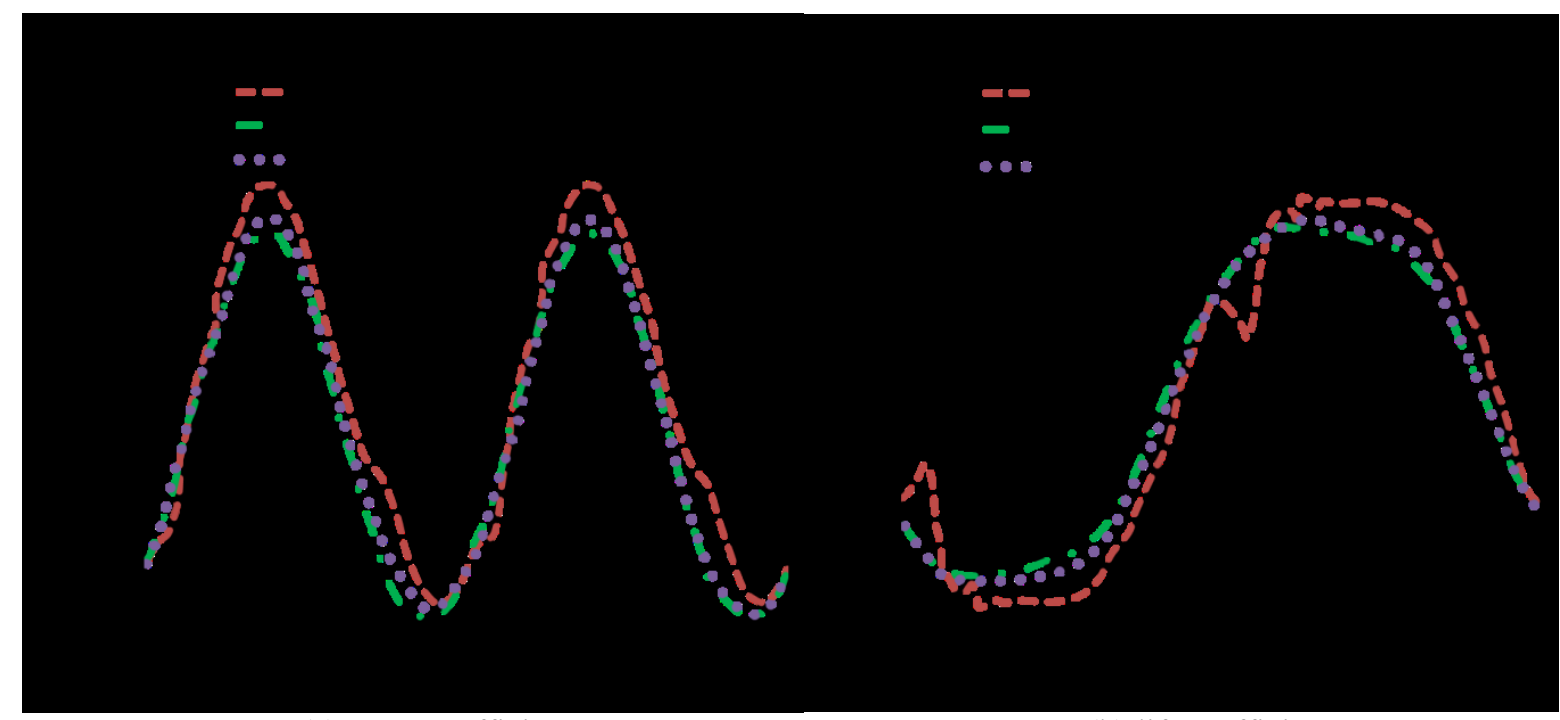

(a) Drag coefficient

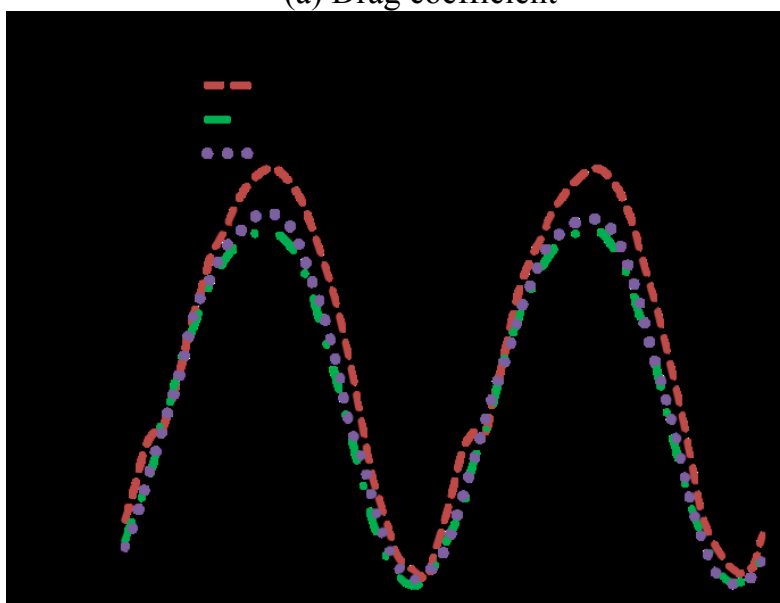

(b) lift coefficient
$\Delta$

\section{(c) power coefficient}

(d) efficiency

Figure 5 Validation on a rigid oscillating foil as an energy extraction device with $h_{0} / c=1.0 ; c_{p i t} / c=1 / 3$; $\boldsymbol{\theta}_{0}=\mathbf{7 5}^{\circ}$ and $\boldsymbol{\varphi}=-\frac{\pi}{2}-$ comparison with Kinsey and Dumas ${ }^{18,33}$ 

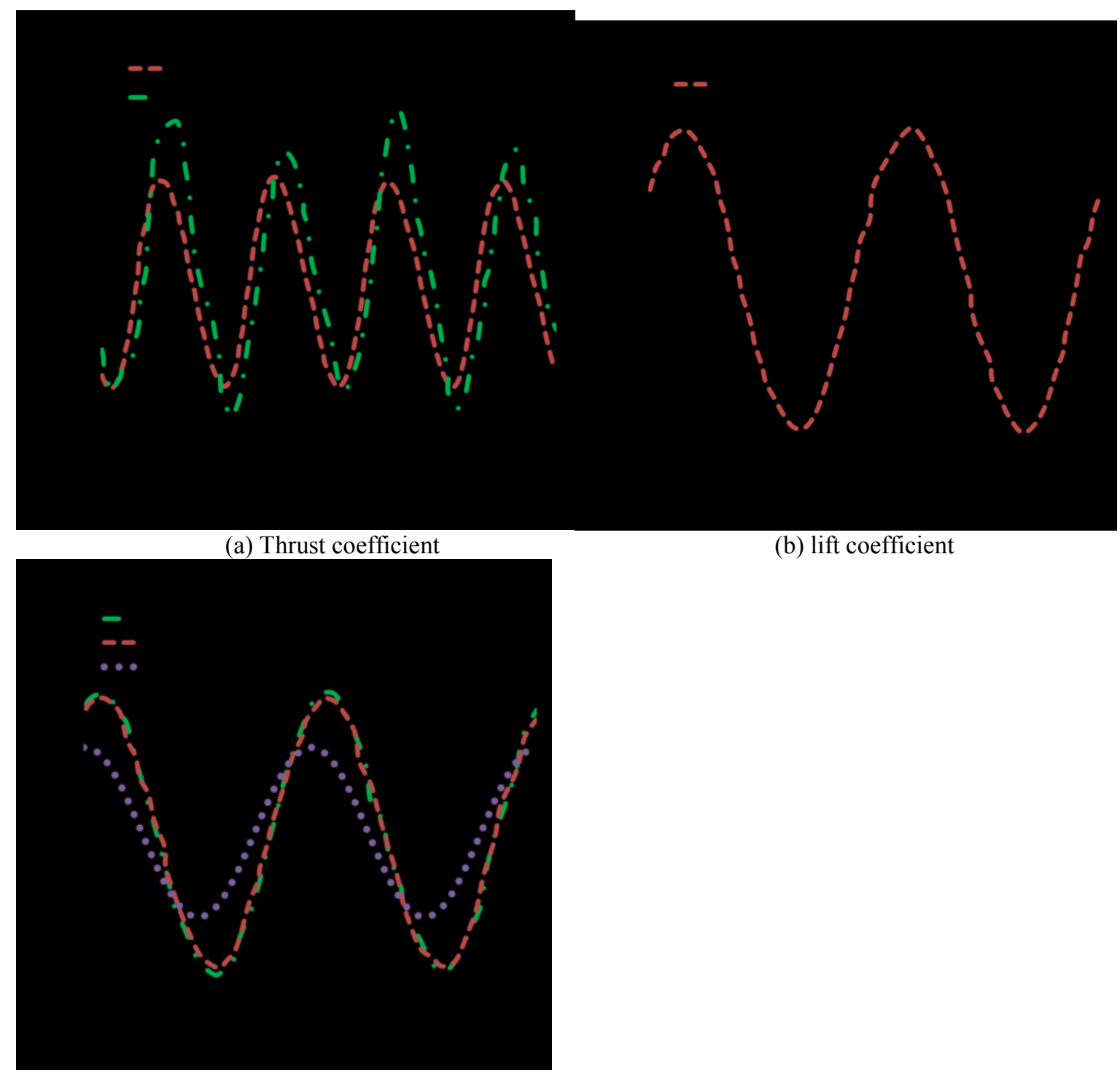

(b) lift coefficient

(c) wing tip edge displacement

Figure 6 Validation on a flexible 3D plunging wing in water flow with $R e=3 \times 10^{4} ; A R=3.0 ; h_{0}=1.75 \times$ $10^{-2} \mathrm{~m} ; f_{0}=1.74 \mathrm{~Hz} ; \Pi=2.14 \times 10^{2} ; E=210 \mathrm{GPa}$ and $D R=7.8$ - comparison with Heathcote et al. ${ }^{21}$ and Gordnier et al. ${ }^{22}$ 


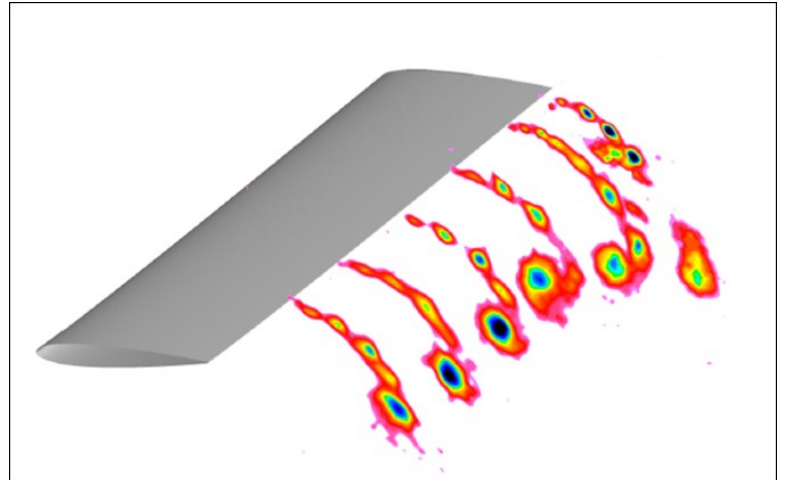

(a) $t / T=0.00$ (Heathcote et al. ${ }^{21}$ experimental result)

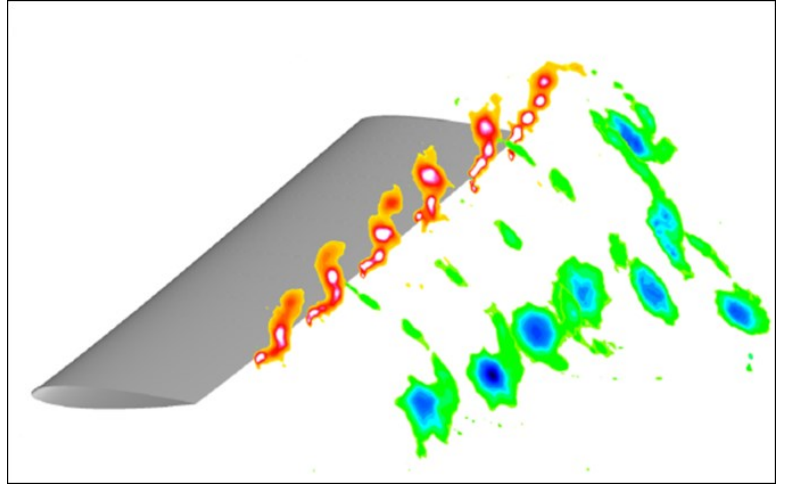

(c) $t / T=0.25$ (Heathcote et al. ${ }^{21}$ experimental result)

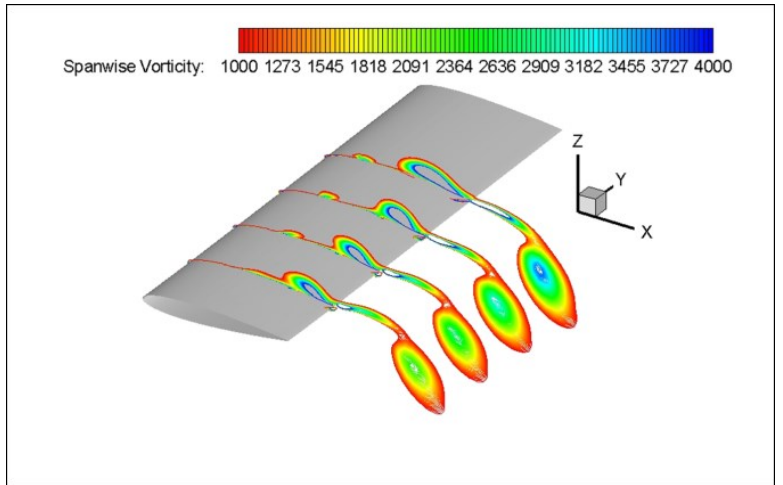

(b) $t / T=0.00$ (present numerical results)

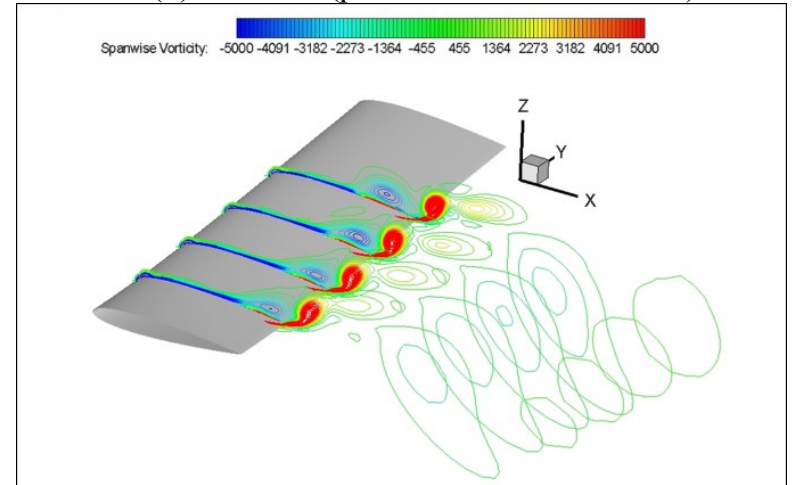

(d) $t / T=0.25$ (present numerical results)

Figure 7 Validation results on spanwise vorticity contour for a flexible $3 D$ wing in $\operatorname{Re}=3 \times 10^{4}$ water flow; $A R=3.0 ; h_{0}=1.75 \times 10^{-2} \mathrm{~m} ; f_{0}=1.74 \mathrm{~Hz} ; \Pi=2.14 \times 10^{2} ; E=210 \mathrm{GPa}$ and $D R=7.8$--Comparison with Heathcote et al. $^{21}$ (a) and (c) Experimental results of Heathcote et al. ${ }^{21}$; (b) and (d) present numerical results 


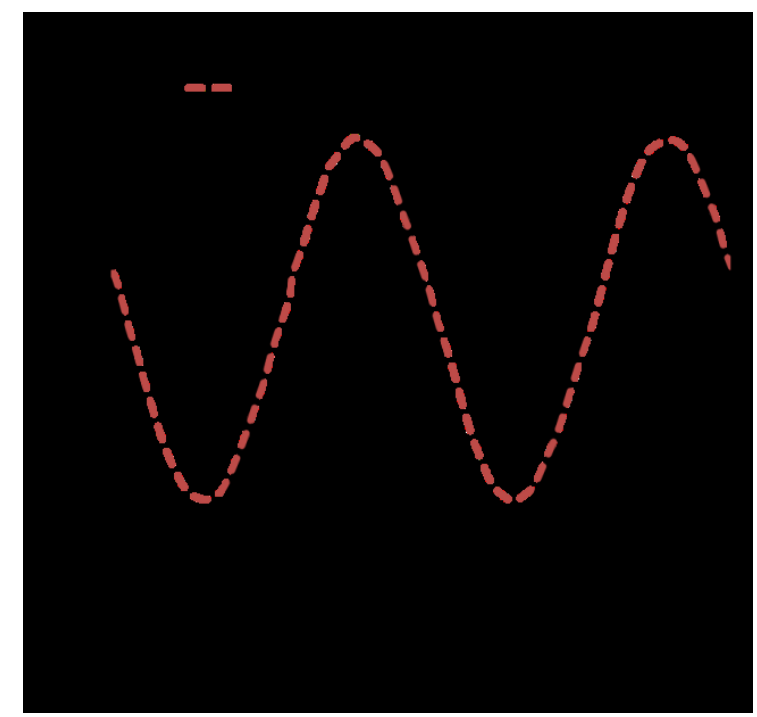

(a) Lift coefficient

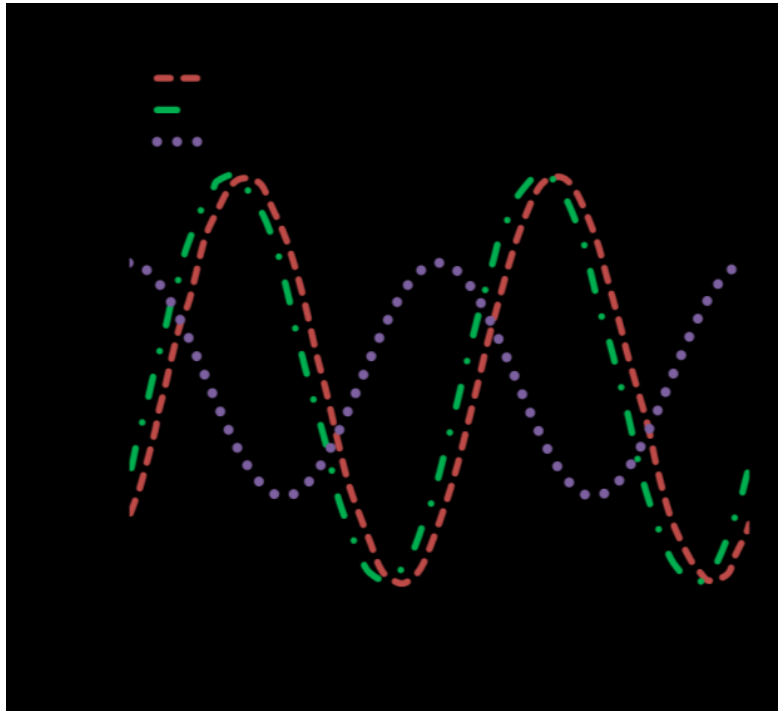

(b) wing tip edge displacement

Figure 8 Validation reuslts on a very flexible 3D plunging wing $\left(R e=3.0 \times 10^{4}\right) A R=3.0 ; h_{0}=1.75 \times 10^{-2}$ $\mathrm{m} ; f_{0}=1.74 \mathrm{~Hz} ; \Pi=4.07 \times 10^{1} ; E=70 \mathrm{GPa}$ and $D R=2.7-$ comparison with Heathcote et al. ${ }^{21}$ and Gordnier et al. ${ }^{24}$ 


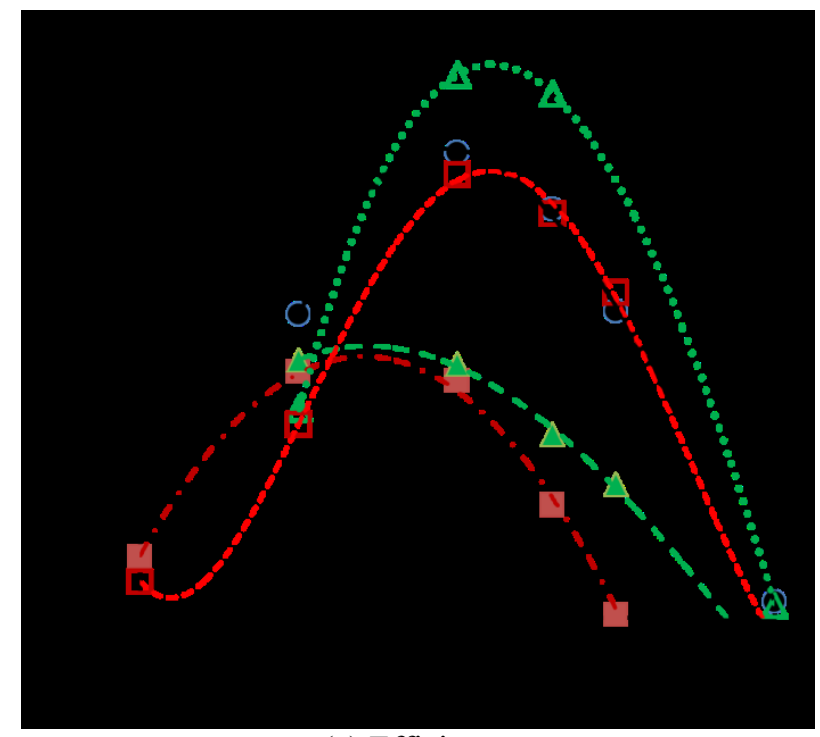

(a) Efficiency

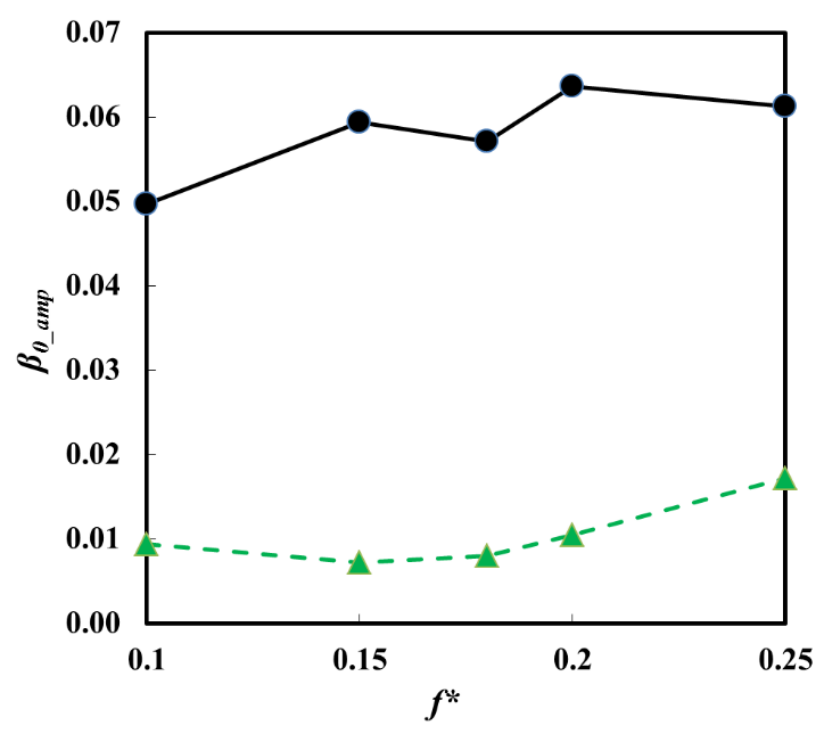

(b) peak trailing edge displacement

Figure 9 The overall performance of a flexible oscillating foil device with $h o / c=1.0 . \bullet \mathrm{Cu} \theta_{0}=60^{\circ} ; \Delta$ WC $\theta_{0}=6^{\circ} ; \square$ Rigid $\theta_{0}=6^{\circ} ; \square$ Rigid $\theta_{0}=75^{\circ} ; \Delta$ WC $\theta_{0}=75^{\circ}$ 


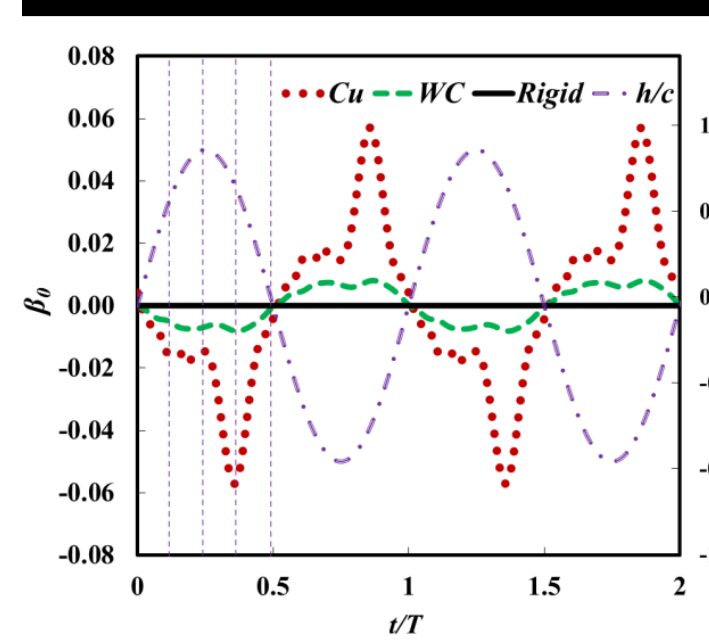

(a) Trailing edge displacement

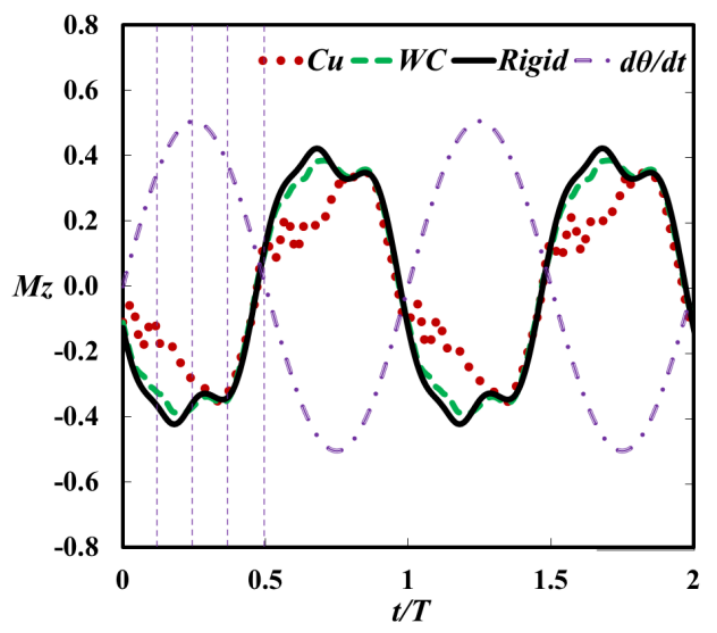

(c) moment coefficient and pitch velocity

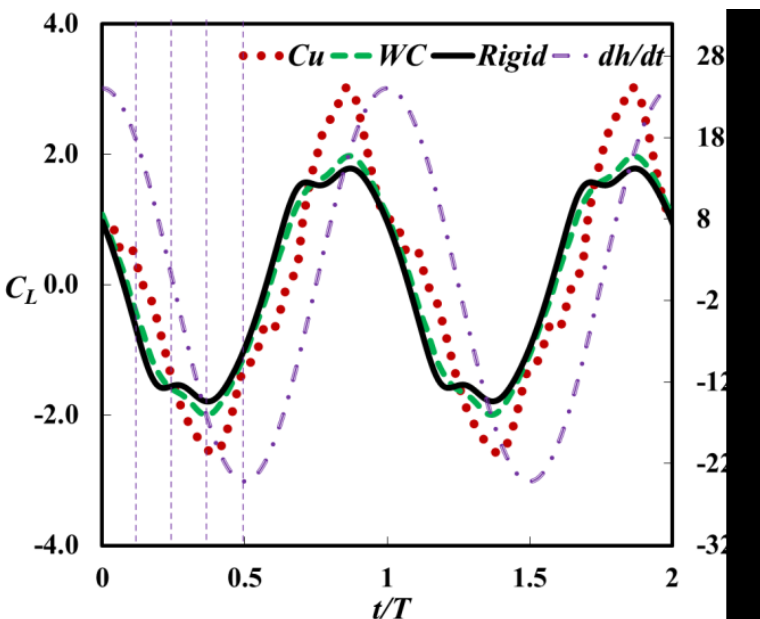

(b) lift coefficient and heaving velocity
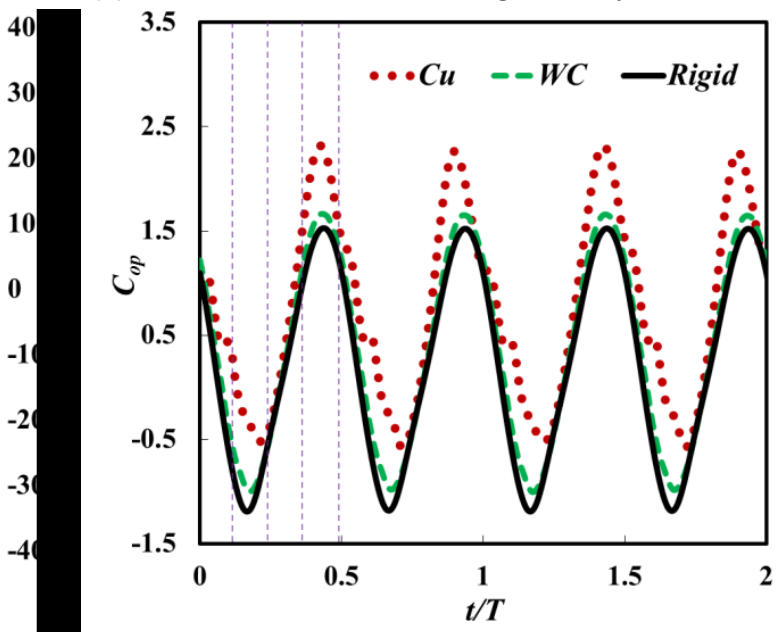

(d) power coefficient

Figure 10 Time histories for foils with $\mathrm{Cu}$ and WC stiffeners at $f^{*}=0.18$ and $\theta_{0}=60^{\circ}$ 


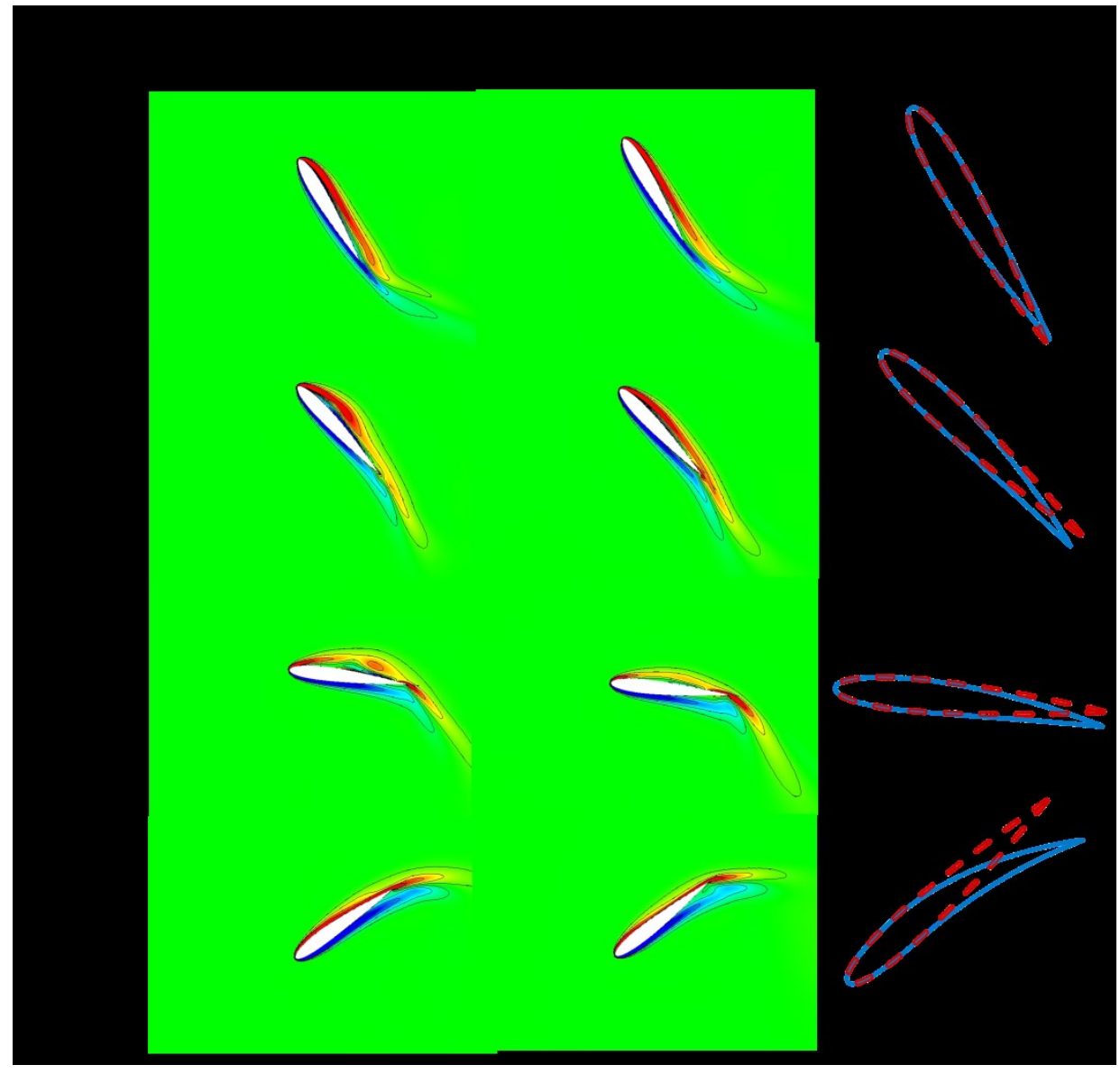

Figure 11 Vorticity contours and schematic representation of foil kinematics for rigid and flexible foils at $f^{*}=0.18 ; \theta_{0}=60^{\circ}$ in half an oscillating period. In the schematic plot, the flexible foil is shown with blue solid lines with a five times scale of original displacement, and the rigid foil is shown as red dashed lines 


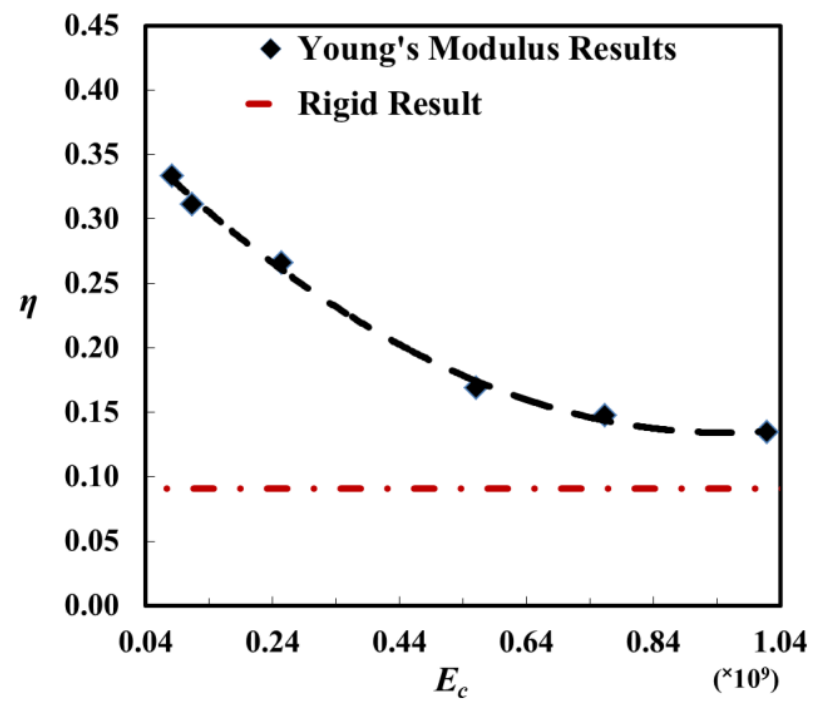

Figure 12 Young's modulus coefficient effect on the efficiency of a flexible foil at $f^{*}=0.18$ and $\theta_{0}=60^{\circ}$ 


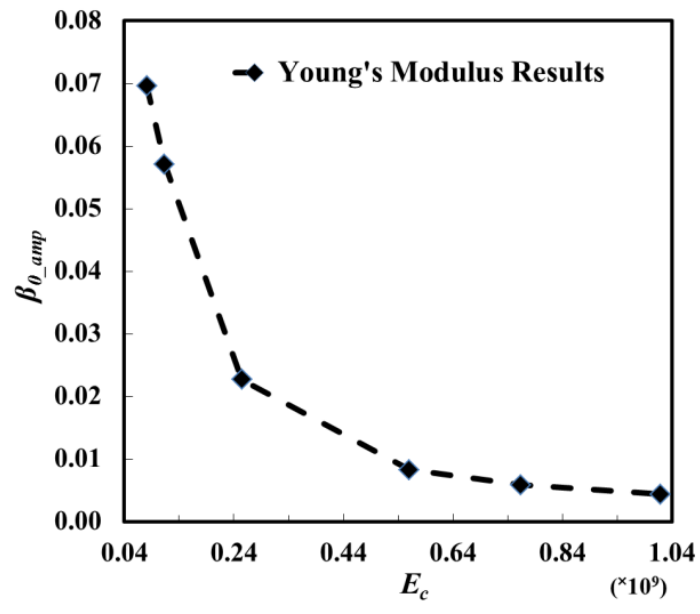

(a) Trailing edge displacement amplitude vs. Young's modulus

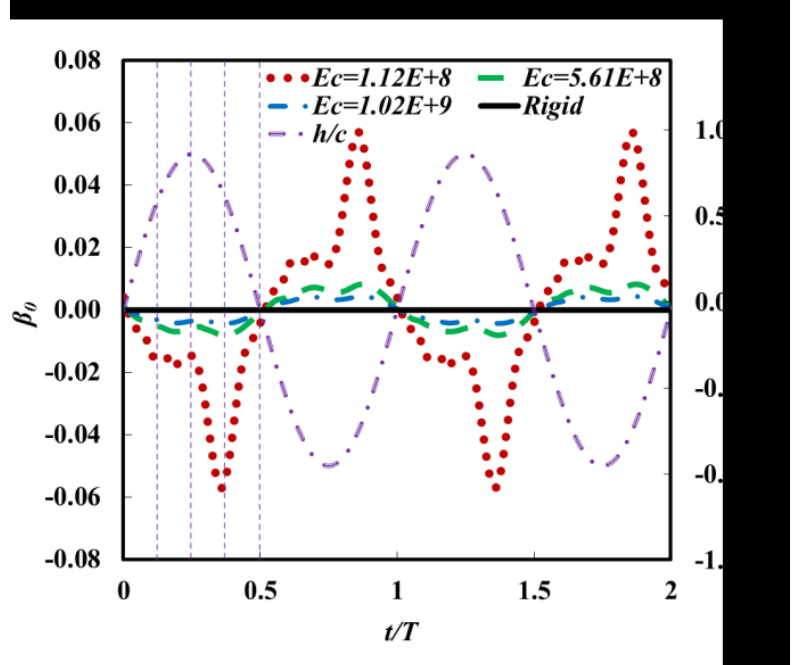

(b) instantaneous trailing edge displacement

Figure 13 Trailing edge deformations of a flexible foil with different Young's modulus coefficient at $f^{*}=0.18$ and $\theta_{0}=60^{\circ}$ 


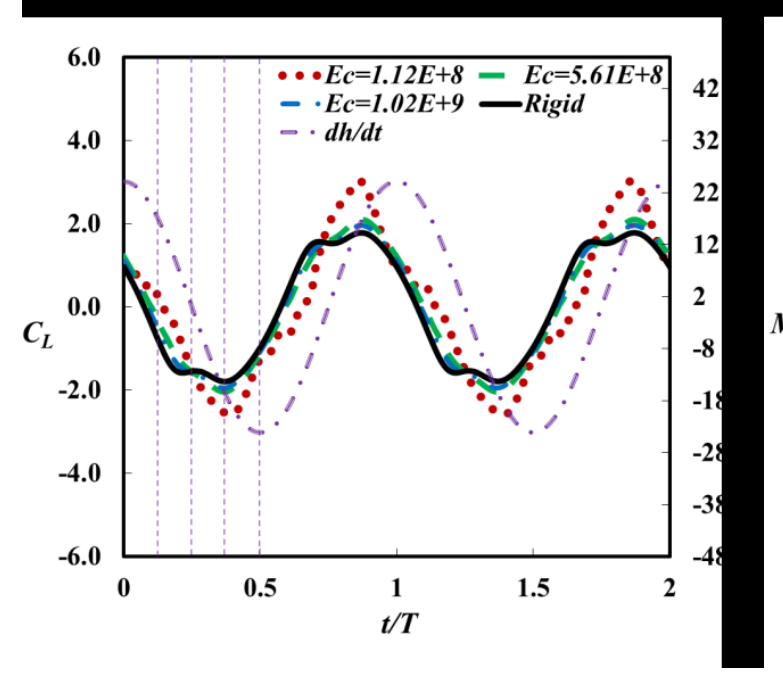

(a) Lift coefficient and heaving velocity

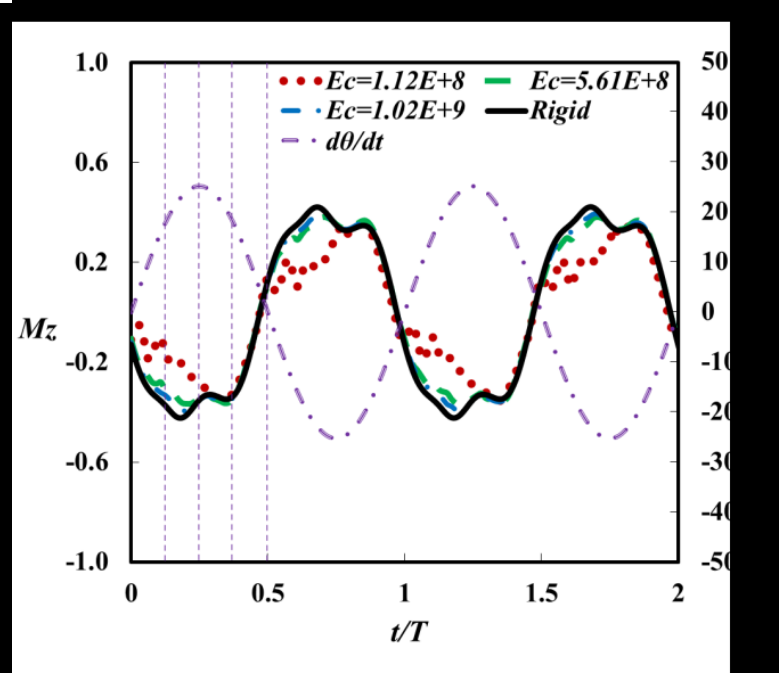

(b) moment coefficient and pitching velocity

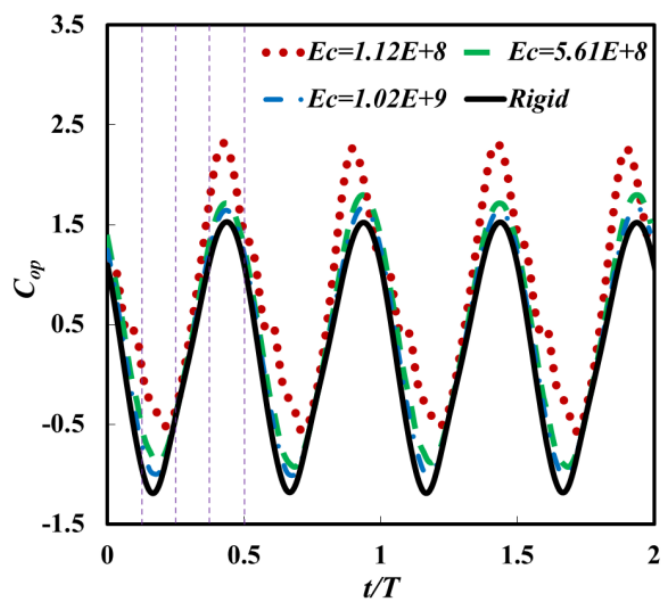

(c) power coefficient

Figure 14 Time histories for Young's modulus coefficient effect at $f^{*}=0.18$ and $\theta_{0}=60^{\circ}$ 


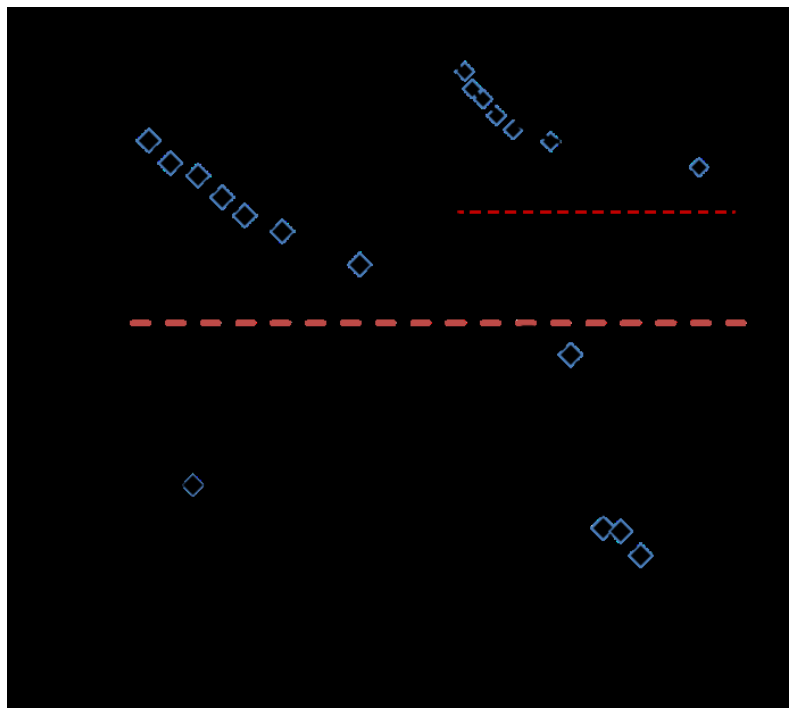

Figure 15 Density ratio effect on the efficiency of a flexible foil at $f^{*}=0.18$ and $\theta_{0}=60^{\circ}$ 

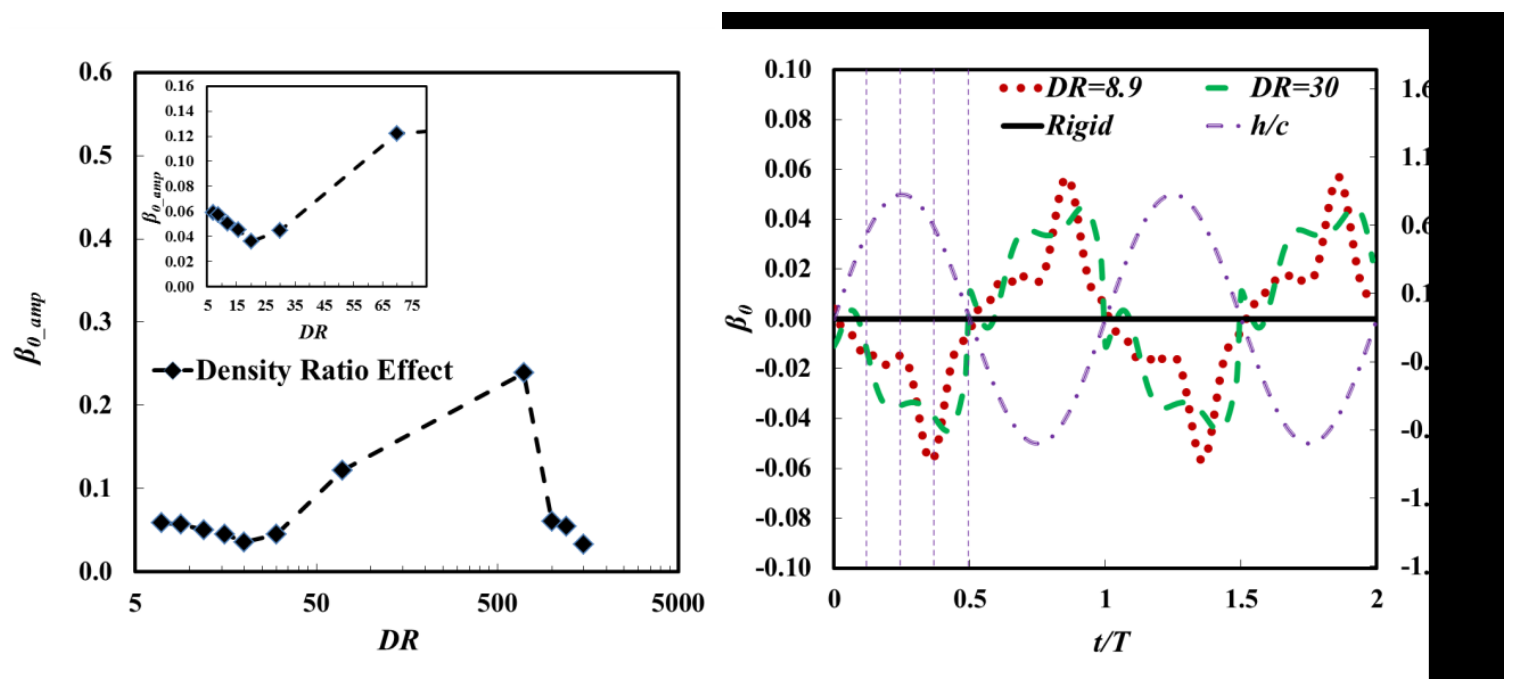

(a) Trailing edge displacement amplitude vs. density ratio (b) instantaneous trailing edge displacement $(D R=8.9$ and 30 , rigid foil)

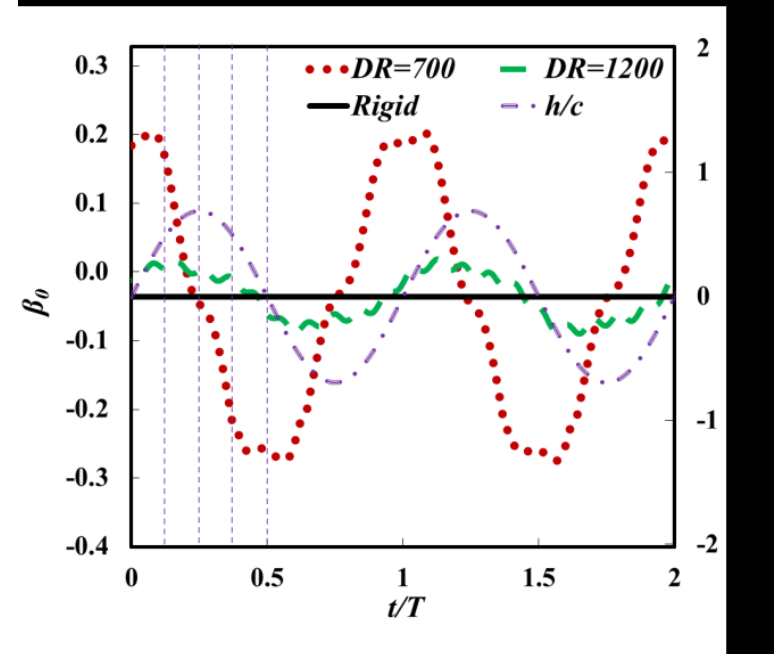

(c) instantaneous trailing edge displacement $(D R=700,1200$ and rigid foil $)$

Figure 16 Density ratio effect at $f^{*}=0.18$ and $\theta_{0}=60^{\circ}$ 


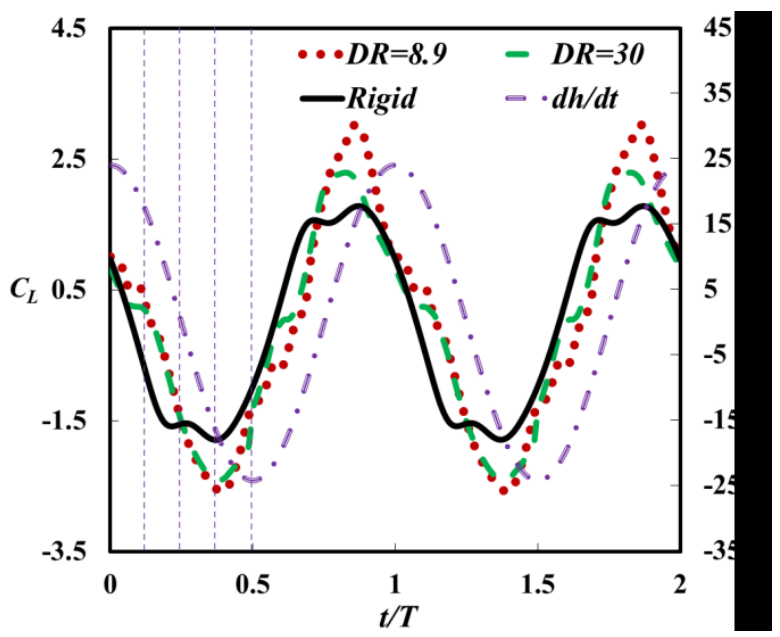

(a) Lift coefficient and heaving velocity

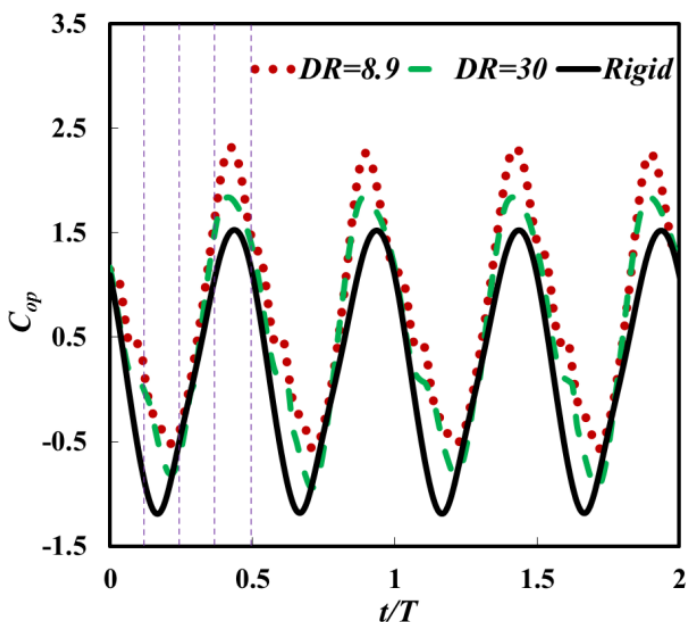

(c) power coefficient

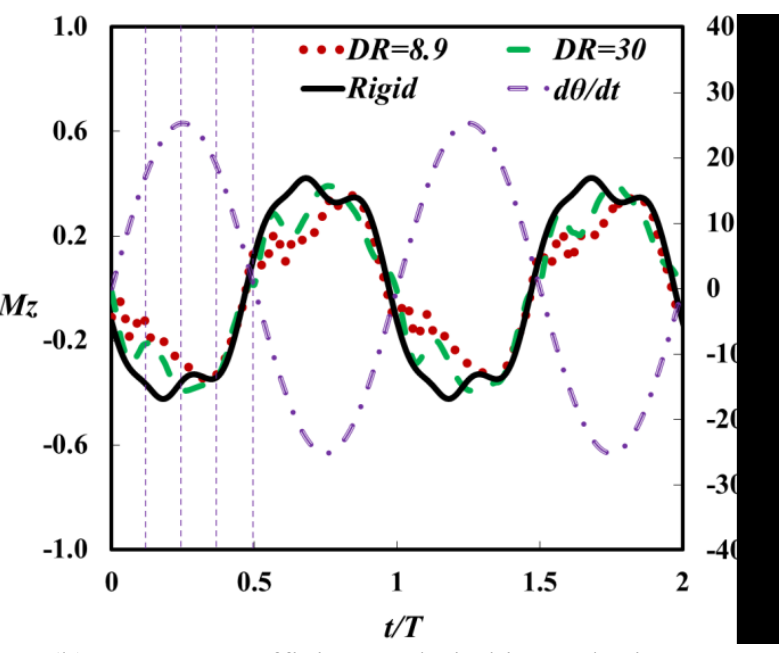

(b) moment coefficient and pitching velocity

Figure 17 Time histories with low density ratios $(D R=8.9$ and 30$)$ at $f^{*}=0.18$ and $\theta_{0}=60^{\circ}$ 


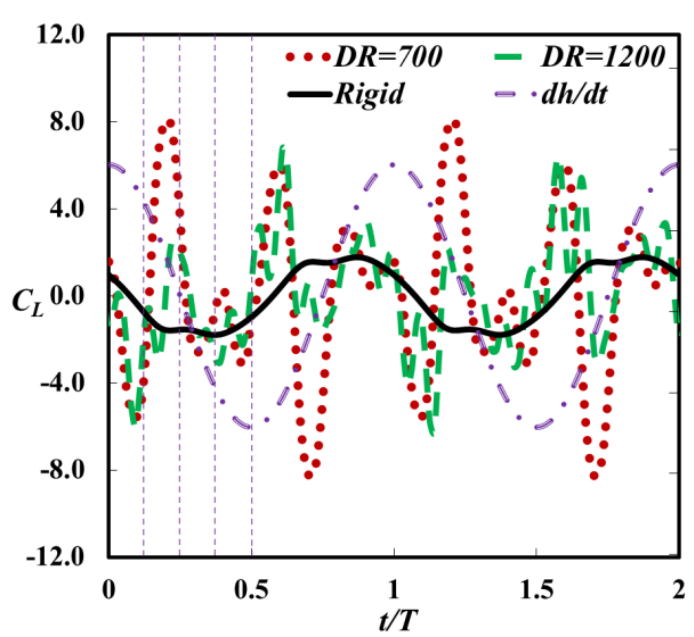

(a) Lift coefficient and heaving velocity

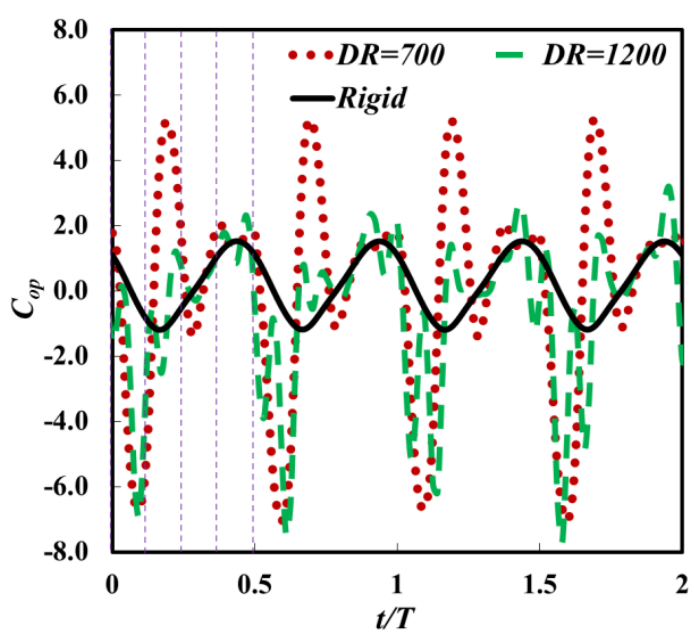

(c) instantaneous power coefficient
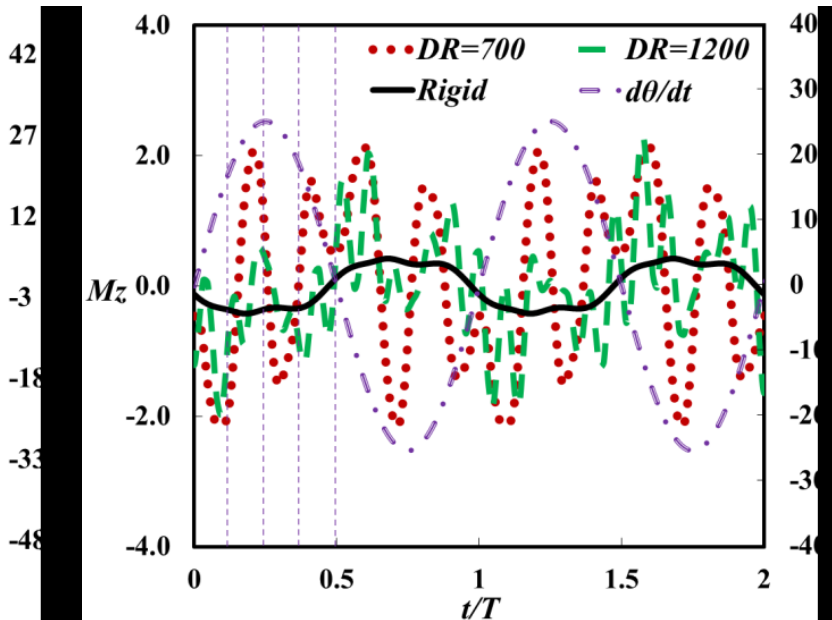

(b) moment coefficient and pitching velocity

Figure 18 Time histories with large density ratios $\left(D R=700\right.$ and 1200) at $f^{*}=0.18$ and $\theta_{0}=60^{\circ}$ 


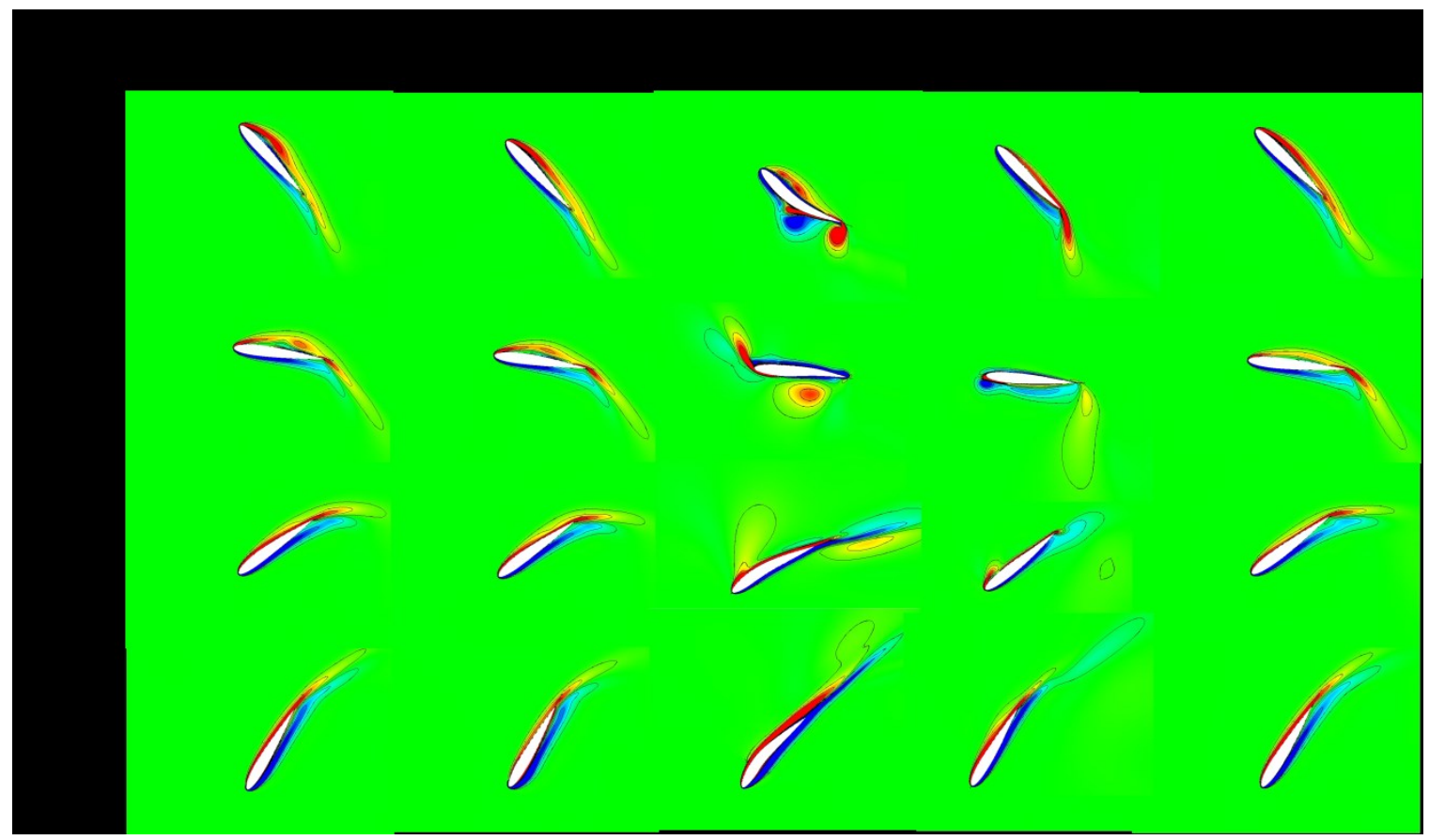

Figure 19 Vorticity contours for density ratio effect at $f^{*}=0.18$ and $\theta_{0}=60^{\circ}$ in half an oscillating period 